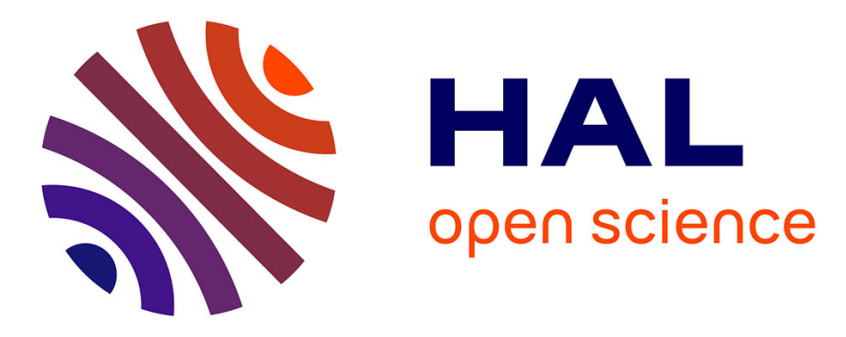

\title{
Stability analysis of networked linear control systems with direct-feedthrough terms
}

Stefan S.H.J. Heijmans, Romain Postoyan, Dragan Nesic, Navid Noroozi, Maurice Heemels

\section{- To cite this version:}

Stefan S.H.J. Heijmans, Romain Postoyan, Dragan Nesic, Navid Noroozi, Maurice Heemels. Stability analysis of networked linear control systems with direct-feedthrough terms. Automatica, 2018, 96, pp.186-200. 10.1016/j.automatica.2018.06.047 . hal-01839461

\section{HAL Id: hal-01839461 https://hal.science/hal-01839461}

Submitted on 14 Jul 2018

HAL is a multi-disciplinary open access archive for the deposit and dissemination of scientific research documents, whether they are published or not. The documents may come from teaching and research institutions in France or abroad, or from public or private research centers.
L'archive ouverte pluridisciplinaire HAL, est destinée au dépôt et à la diffusion de documents scientifiques de niveau recherche, publiés ou non, émanant des établissements d'enseignement et de recherche français ou étrangers, des laboratoires publics ou privés. 


\title{
Stability Analysis of Networked Linear Control Systems with Direct-Feedthrough Terms *
}

\author{
S.H.J. Heijmans ${ }^{a}$, R. Postoyan ${ }^{b}$, D. Nešićc ${ }^{c}$, N. Noroozi ${ }^{\text {d }}$, W.P.M.H. Heemels ${ }^{\text {a }}$ \\ ${ }^{a}$ Eindhoven University of Technology, Department of Mechanical Engineering, 5600 MB, Eindhoven, The Netherlands \\ ${ }^{\mathrm{b}}$ Université de Lorraine, CRAN, UMR, 7039 and CNRS, CRAN, UMR 7039, France \\ ${ }^{\mathrm{c}}$ The University of Melbourne, Department of Electrical and Electronic Engineering, Parkville 3010, Victoria, Australia \\ ${ }^{\mathrm{d}}$ University of Passau, Faculty of Computer Science and Mathematics, Innstraße 33, 94032 Passau, Germany
}

\begin{abstract}
We consider networked control systems (NCSs) composed of a linear plant and a linear controller interconnected by packetbased communication channels with communication constraints. We are interested in the setup where direct-feedthrough terms are present in the plant and/or in the controller, a case that is largely ignored in the literature due to its inherent complexity and counterintuitive results in the analysis despite its relevance for important classes of controllers including ProportionalIntegral (PI) regulators. This setup calls for a novel stability analysis, for which we take a renewed look at the concept of uniformly globally exponentially stable (UGES) scheduling protocols that turned out to be instrumental in earlier approaches. We provide a generalization of the UGES property, called $\left(D_{\mathbf{P}}, D_{\mathbf{C}}\right)$-UGES with $D_{\mathbf{P}} / D_{\mathbf{C}}$ being the direct-feedthrough matrices of the plant/controller, respectively, and we present generic conditions on these direct-feedthrough terms $D_{\mathbf{P}} / D_{\mathbf{C}}$ such that the classical UGES property of scheduling protocols implies $\left(D_{\mathbf{P}}, D_{\mathbf{C}}\right)$-UGES. This allows us to derive conditions leading to a maximally allowable transmission interval (MATI) such that stability of the overall NCS is guaranteed. In addition, it is shown that it is possible to get more tailored results for the well-known sampled-data (SD), round-robin (RR), and try-oncediscard (TOD) protocols leading to less conservative conditions on the direct-feedthrough terms than the generic ones. We also introduce new $\left(D_{\mathbf{P}}, D_{\mathbf{C}}\right)$-UGES scheduling protocols, designed to handle the direct-feedthrough terms in a more effective way than existing protocols. Our results are illustrated using the example of a batch reactor.
\end{abstract}

Key words: Networked control systems; Lyapunov methods; Scheduling protocols; Exponential stability.

\section{Introduction}

In many control applications, including manufacturing plants, vehicles, and aircraft, communication is needed for the exchange of information and control signals between spatially distributed system components, such as supervisory computers, controllers, sensors, and actua-

\footnotetext{
* A preliminary version of this paper was presented at the IEEE $55^{\text {th }}$ Conference on Decision and Control, December 12-14, 2016, Las Vegas, NV, USA.

Stefan Heijmans and Maurice Heemels are supported by the Innovational Research Incentives Scheme under the VICI grant "Wireless control systems: A new frontier in automation" (No. 11382) awarded by STW (Dutch Science Foundation) and NWO (The Netherlands Organization for Scientific Research), Romain Postoyan is partially supported by the ANR under the grant COMPACS (ANR-13-BS03-0004-02), and Dragan Nešić is supported by the Australian Research Council under the Discovery Project DP170104099.

Email address corresponding author:

s.h.j.heijmans@tue.nl (S.H.J. Heijmans).
}

tors. When sensor and actuator data is communicated over a shared (wired or wireless) packet-based communication network, the system is called a networked control system (NCS). Such NCSs have received considerable attention in recent years $[9,18,32,34]$. This interest is motivated by the many advantages their flexible architectures offer, such as reduced weight, volume and installation costs, and better maintainability, when compared to conventional control systems in which sensor and actuation data is transmitted over dedicated point-to-point (wired) links, see, e.g., [27]. Additionally, wireless communication is able to overcome the physical limitations of employing wired links, which is very appealing in, for instance, intelligent transportation, see, e.g., [25], and remote surgery, see, e.g., [22]. On the other hand, the usage of packet-based networked communication comes also with the inevitable network-induced imperfections, such as varying delays, dropouts, varying transmission intervals, and so on. Moreover, as the communication network is often shared by multiple sensors and actuators, there is a need for so-called scheduling protocols, which govern the access of the nodes to the network. 
To deal with all these network-induced phenomena, novel design and analysis approaches are required. A popular design approach for NCSs herein is the so-called emulation method, see, e.g., [5, 14, 23, 26, 30]. The idea is to first design a continuous-time controller for the continuous-time plant while ignoring the communication constraints. Then, the controller is implemented via the packet-based communication network with its scheduling protocol and it is shown that stability properties are preserved when information is transmitted frequently enough. By using the concept of uniformly globally exponentially stable (UGES) scheduling protocols introduced in [23], conditions leading to the determination of so-called maximal allowable transmission intervals (MATIs) guaranteeing overall stability or $\mathcal{L}_{p}$-gain performance of the NCS have been derived, see [5, 23]. In addition to this general setup, many extensions can be found in $[7,12,14,15]$, and the references therein.

It is interesting to observe that none of the aforementioned results in $[5,7,12,14,15,23,26,30]$ considered the inclusion of so-called direct-feedthrough terms, i.e., terms that allow a direct connection between the control input and the plant output (and vice versa) and that are essential to model classical controllers commonly used in the industry such as Proportional-Integral(-Derivative) $(\mathrm{PI}(\mathrm{D}))$ regulators and state feedback controllers, when both actuator and sensor signals are transmitted over the communication network. However, as we will show in this paper, this is not surprising as these direct-feedthrough terms lead to nontrivial difficulties in terms of modeling and analysis. In particular, the presence of the directfeedthrough terms modifies the model of the networkinduced error at transmissions making it, in contrast to $[5,7,12,14,15,23,26,30]$, dependent on plant and controller parameters. This complicates the analysis significantly and leads to various counterintuitive results, as will be highlighted throughout the paper. As a result, a novel (stability) analysis is needed to address standard (UGES) scheduling protocols such as the sampled-data (SD) (which updates all network nodes simultaneously), round-robin (RR) (which assigns access to the network in a cyclic manner), and try-once-discard (TOD) (which gives access to the node with the largest error) protocols.

Given the importance of PI(D) control and other control/plant structures with direct-feedthrough terms, we already addressed this so-called direct-feedthrough problem in our preliminary works [24] and [16]. In particular, it was shown in [24] that, for the case where only the controller contained direct-feedthrough terms, stability of nonlinear NCSs could still be guaranteed when using standard scheduling protocols such as the SD and RR protocols. In [16], linear NCSs with direct-feedthrough terms in both the plant and the controller were studied, which introduces additional difficulties. It was shown that for the standard SD, RR, and TOD protocols, under certain conditions, the direct-feedthrough terms can be incorporated in the NCS stability analysis of $[5,23,30]$.
In this paper, we build upon our preliminary work [16] and again consider linear NCSs with direct-feedthrough terms in both the plant and the controller. We provide a generalization of the UGES property for scheduling protocols, called $\left(D_{\mathbf{P}}, D_{\mathbf{C}}\right)$-UGES, where $D_{\mathbf{P}}$ and $D_{\mathbf{C}}$ are the direct-feedthrough matrices of the plant and controller, respectively, and we present generic conditions on these direct-feedthrough terms $D_{\mathbf{P}}$ and $D_{\mathbf{C}}$ such that any UGES protocol in the classical sense is also $\left(D_{\mathbf{P}}, D_{\mathbf{C}}\right)$-UGES, which is important to apply the stability analysis of $[5,23,30]$ to guarantee stability of the NCS. Moreover, it is shown that for the SD, RR, and TOD protocols these conditions can be made less conservative by exploiting the knowledge we have about the structure of the protocol. This will also lead to the counterintuitive result of the "smarter" TOD protocol not always being better than the RR protocol and that updating all the nodes simultaneously (exploiting the SD protocol) can be worse than updating the nodes one by one. Finally, we introduce new $\left(D_{\mathbf{P}}, D_{\mathbf{C}}\right)$-UGES scheduling protocols, designed to handle the direct-feedthrough terms in a more effective way than the existing protocols in the case we do not have so-called mixed nodes, i.e., in the case that we only have nodes that are only related to the actuators and/or nodes that are only related to the sensors. These novel results significantly extend our preliminary work [16] as, in this paper, we provide full derivations and proofs, provide generic conditions for any UGES protocol to be $\left(D_{\mathbf{P}}, D_{\mathbf{C}}\right)$-UGES and not only for the SD, RR, and TOD protocols as in [16], introduce new $\left(\left(D_{\mathbf{P}}, D_{\mathbf{C}}\right)\right.$-UGES) scheduling protocols (called the $\mathrm{SD}+$, the periodic switching, and maximal error switching scheduling protocol), and present various counterintuitive results, which, for instance, show that being "smarter" is not always the best solution. To illustrate our results we apply them to the benchmark example of a batch reactor.

The remainder of this paper is organized as follows. After presenting the necessary notation, the class of systems considered in this paper is described in Section 2 including a suitable hybrid model for the NCS. In Section 3, we briefly recapitulate the stability analysis of NCSs of $[5,23]$, although slightly adapted to take into account the presence of the direct-feedthrough terms. In Section 4, we revise the concept of UGES scheduling protocols and provide generic conditions on the directfeedthrough terms such that the stability analysis presented in Section 3 can be applied. These conditions are then improved for the well-known protocols SD, RR, and TOD in Section 5 and new scheduling protocols are introduced in Section 6. Finally, in Section 7, the batch reactor example illustrating our results is provided, and in Section 8 concluding remarks are given. All of the proofs are provided in Appendix A.

Notation: The set of real numbers is denoted by $\mathbb{R}:=(-\infty, \infty)$ and the sets of non-negative real numbers and integers by $\mathbb{R}_{\geq 0}:=[0, \infty)$ and $\mathbb{N}:=0,1,2, \ldots$, re- 
spectively. For vectors $v_{1}, v_{2}, \ldots, v_{n} \in \mathbb{R}^{n}$, we denote by $\left(v_{1}, v_{2}, \ldots, v_{n}\right)$ the vector $\left[\begin{array}{llll}v_{1}^{\top} & v_{2}^{\top} \ldots & v_{n}^{\top}\end{array}\right]^{\top}$, and by $|\cdot|$ and $\langle\cdot, \cdot\rangle$ the Euclidean norm and the usual inner product, respectively. We use the notation $r^{+}=r\left(t^{+}\right)=\lim _{\tau \downarrow t} r(\tau)$ where $r$ is any left-continuous mapping from $\mathbb{R}$ to $\mathbb{R}^{n}$. The $n$ by $n$ identity and zero matrices are denoted by $I_{n}$ and $0_{n}$, respectively. When the dimensions are clear from the context, these notations are simplified to $I$ and 0 . For a symmetric matrix $A \in \mathbb{R}^{n \times n}, \lambda_{\min }(A) / \lambda_{\max }(A)$ denote the smallest/largest eigenvalue of $A$. A function $f: \mathbb{R}^{m} \rightarrow \mathbb{R}^{n}$ is said to be globally Lipschitz there exists $M>0$ such that for all $x, y \in \mathbb{R}^{m}$ it holds that $|f(x)-f(y)| \leq M|x-y|$.

\section{System description: The NCS model}

In this section, the considered class of systems is introduced, where we in particular focus on the influence and impact of the direct-feedthrough terms on the NCS configuration as introduced in the literature, see also [16].

\subsection{Networked control configuration}

In this work, we consider the NCS as shown in Fig. 1, where the continuous-time plant $\mathcal{P}$ is given by

$$
\mathcal{P}:\left[\begin{array}{c}
\dot{x}_{p} \\
y
\end{array}\right]=\left[\begin{array}{ll}
A_{\mathbf{P}} & B_{\mathbf{P}} \\
C_{\mathbf{P}} & D_{\mathbf{P}}
\end{array}\right]\left[\begin{array}{c}
x_{p} \\
\hat{u}
\end{array}\right],
$$

where $x_{p} \in \mathbb{R}^{m_{x_{p}}}$ denotes the state, $\hat{u} \in \mathbb{R}^{m_{u}}$ the most recently received control input, and $y \in \mathbb{R}^{m_{y}}$ the measured output to the controller.

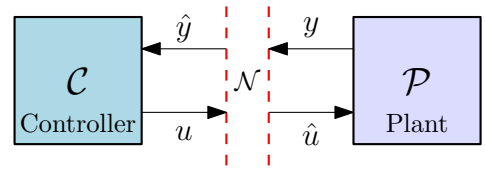

Fig. 1. The NCS setup.

As shown in Fig. 1, the plant $\mathcal{P}$ is controlled by the controller $\mathcal{C}$, and they communicate with each other via the network $\mathcal{N}$. The controller $\mathcal{C}$ itself is described by

$$
\mathcal{C}:\left[\begin{array}{l}
\dot{x}_{c} \\
u
\end{array}\right]=\left[\begin{array}{ll}
A_{\mathbf{C}} & B_{\mathbf{C}} \\
C_{\mathbf{C}} & D_{\mathbf{C}}
\end{array}\right]\left[\begin{array}{c}
x_{c} \\
\hat{y}
\end{array}\right],
$$

where $x_{c} \in \mathbb{R}^{m_{c}}$ denotes the controller state, $\hat{y} \in \mathbb{R}^{m_{y}}$ the most recently received output measurement of the plant, and $u \in \mathbb{R}^{m_{u}}$ the control input. Note now that the difference between the setups in $[5,7,12,14,15,23,30]$ and (1)-(2) is given by the direct-feedthrough matrices $D_{\mathbf{P}}$ and $D_{\mathbf{C}}$ from which at least one of them is nonzero.

To complete the description of the NCS setup, we first explain how the communication network $\mathcal{N}$ operates. This network $\mathcal{N}$ has a collection of sampling/ transmission times $t_{j}, j \in \mathbb{N}$, which satisfy $0 \leq t_{1}<t_{2}<\cdots$. At such a transmission time $t_{j}$, (parts of) the output $y$ and the input $u$ are sampled and transmitted over the network $\mathcal{N}$ to the controller $\mathcal{C}$ and the plant $\mathcal{P}$, respectively.

In the considered setup, similar to $[5,12,14,15,23,30]$, it is assumed that the transmission times satisfy

$$
\delta \leq t_{j+1}-t_{j} \leq \tau_{\text {mati }}
$$

for all $j \in \mathbb{N}$, where $\tau_{\text {mati }}$ denotes the maximally allowable transmission interval (MATI) and where $0<\delta \leq \tau_{\text {mati }}$ is a positive time between two consecutive transmission times. It should be noted that, theoretically, $\delta$ can be taken arbitrarily small since it is only imposed to prevent Zeno behavior [20], but in each example it will be strictly positive and determined by hardware limitations.

In addition to this sequence of transmission times, the network $\mathcal{N}$ might also be subdivided in several (sensor and/or actuator) nodes, where each node corresponds to a subset of the entries $y / \hat{y}$ and/or $u / \hat{u}$. As such, there is the need for a scheduling protocol that determines which of the nodes in the network is granted access to the network at a transmission time, see also [5, 14, 23]. After a node is granted access to the network, it collects and transmits the values of the corresponding entries in $y\left(t_{j}\right)$ and $u\left(t_{j}\right)$, which results in an update according to

$$
\begin{aligned}
& \hat{y}\left(t_{j}^{+}\right)=y\left(t_{j}\right)+h_{y}\left(j, e\left(t_{j}\right)\right) \\
& \hat{u}\left(t_{j}^{+}\right)=u\left(t_{j}\right)+h_{u}\left(j, e\left(t_{j}\right)\right),
\end{aligned}
$$

where the function $h:=\left(h_{y}, h_{u}\right)$ (with slight abuse of notation) models the scheduling protocol and where $e$ denotes the network-induced error defined by

$$
e:=\left[\begin{array}{l}
e_{y} \\
e_{u}
\end{array}\right]=\left[\begin{array}{l}
\hat{y}-y \\
\hat{u}-u
\end{array}\right] .
$$

Note that we follow here the same method for modeling the scheduling protocols as described in $[5,14,23]$ based on the protocol function $h: \mathbb{N} \times \mathbb{R}^{m_{e}} \rightarrow \mathbb{R}^{m_{e}}$, which can be used to describe, e.g., the SD, RR, and TOD protocols, see also Section 5 below. Indeed, in (4) it is determined on the basis of the transmission counter $j$ and the network-induced error $e\left(t_{j}\right)$ which node is allowed to communicate and typically the corresponding entries in $h$ are zero, see $[5,14,23]$ for a detailed description. Finally, it is assumed that $\hat{y}$ and $\hat{u}$ are constant in between two successive transmissions (zero-order-hold $(\mathrm{ZOH})$ ). However, this can easily be modified if desired, see [23].

From this point forward, we will also use the shorthand notations $\hat{y}^{+}=\hat{y}\left(t_{j}^{+}\right), \hat{u}^{+}=\hat{u}\left(t_{j}^{+}\right), y=y\left(t_{j}\right), \hat{y}=\hat{y}\left(t_{j}\right)$, $u=u\left(t_{j}\right), \hat{u}=\hat{u}\left(t_{j}\right), x_{p}=x_{p}\left(t_{j}\right), x_{c}=x_{c}\left(t_{j}\right), e^{+}=e\left(t_{j}^{+}\right)$, and $e=e\left(t_{j}\right)$.

\subsection{Updating the network-induced error e}

Because of the presence of the direct-feedthrough matrices $D_{\mathbf{P}}$ and $D_{\mathbf{C}}$ in the networked interconnection, we 
have that $u$ and $y$ depend on the networked values $\hat{y}$ and $\hat{u}$, respectively. As a result, an update of $\hat{y}$ and $\hat{u}$ also results in a change of the values of $y$ and $u$, i.e., we have that, in view of (1)-(2),

$$
y^{+}=C_{\mathbf{P}} x_{p}+D_{\mathbf{P}} \hat{u}^{+} \text {and } u^{+}=C_{\mathbf{C}} x_{c}+D_{\mathbf{C}} \hat{y}^{+} .
$$

As a consequence, we encounter difficulties regarding the modeling of the update equation for the networkinduced error $e$. To put this into more context, consider the following analysis. Using the expressions of (1)-(2) and (5) it can be obtained that the errors $e_{y}$ and $e_{u}$ themselves are given by

$$
\begin{aligned}
& e_{y}=\hat{y}-y=\hat{y}-C_{\mathbf{P}} x_{p}-D_{\mathbf{P}} \hat{u} \\
& e_{u}=\hat{u}-u=\hat{u}-C_{\mathbf{C}} x_{c}-D_{\mathbf{C}} \hat{y} .
\end{aligned}
$$

Consider now the situation that we have an update of our networked values at transmission time $t_{j}, j \in \mathbb{N}$, according to (4), i.e.,

$$
\begin{aligned}
& \hat{y}^{+}=y+h_{y}(j, e)=C_{\mathbf{P}} x_{p}+D_{\mathbf{P}} \hat{u}+h_{y}(j, e) \\
& \hat{u}^{+}=u+h_{u}(j, e)=C_{\mathbf{C}} x_{c}+D_{\mathbf{C}} \hat{y}+h_{u}(j, e) .
\end{aligned}
$$

By using (6)-(8), we derive that this update of the networked values leads to the network-induced error being updated according to

$$
\begin{aligned}
e_{y}^{+} & =\hat{y}^{+}-C_{\mathbf{P}} x_{p}-D_{\mathbf{P}} \hat{u}^{+} \\
& =D_{\mathbf{P}} \hat{u}+h_{y}(j, e)-D_{\mathbf{P}} C_{\mathbf{C}} x_{c}-D_{\mathbf{P}} D_{\mathbf{C}} \hat{y}-D_{\mathbf{P}} h_{u}(j, e) \\
& =h_{y}(j, e)-D_{\mathbf{P}} h_{u}(j, e)+D_{\mathbf{P}} e_{u} \\
e_{u}^{+} & =\hat{u}^{+}-C_{\mathbf{C}} x_{c}-D_{\mathbf{C}} \hat{y}^{+} \\
& =D_{\mathbf{C}} \hat{y}+h_{u}(j, e)-D_{\mathbf{C}} C_{\mathbf{P}} x_{p}-D_{\mathbf{C}} D_{\mathbf{P}} \hat{u}+D_{\mathbf{C}} h_{y}(j, e) \\
& =h_{u}(j, e)-D_{\mathbf{C}} h_{y}(j, e)+D_{\mathbf{C}} e_{y} .
\end{aligned}
$$

Hence, we have that, in general, the update equation of the error $e$ can be described by using an update function $h_{d f}: \mathbb{R}^{m_{e}} \rightarrow \mathbb{R}^{m_{e}}$, i.e.,

$$
e^{+}=h(j, e)+\underbrace{\left[\begin{array}{cc}
0_{m_{y}} & D_{\mathbf{P}} \\
D_{\mathbf{C}} & 0_{m_{u}}
\end{array}\right](e-h(j, e))}_{=: h_{d f}(j, e)} .
$$

This shows that the update of the error $e$ essentially differs from the situation without direct-feedthrough terms (i.e., $D_{\mathbf{P}}=0$ and $D_{\mathbf{C}}=0$ ), as in $[5,7,12,14,15,23,30]$, which resulted in

$$
e^{+}=h(j, e) .
$$

As such, a careful reconsideration is needed regarding the analysis of the NCSs described by (1)-(4). To this end, we start by modeling the NCS in the form of a hybrid system [11].

\subsection{A hybrid modeling framework}

Based on the above setup, the triple $(\mathcal{P}, \mathcal{C}, \mathcal{N})$ can be rewritten into the format of a hybrid system $\mathcal{H}$, as described in $[5,23,30]$, where each jump of the hybrid system corresponds to an update of the networked values according to (4). To do so, based on (1), (2), and (5), we write

$$
\begin{aligned}
\hat{u} & =u+e_{u}=C_{\mathbf{C}} x_{c}+D_{\mathbf{C}} \hat{y}+e_{u} \\
& =C_{\mathbf{C}} x_{c}+D_{\mathbf{C}}\left(y+e_{y}\right)+e_{u} \\
& =C_{\mathbf{C}} x_{c}+D_{\mathbf{C}}\left(C_{\mathbf{P}} x_{p}+D_{\mathbf{P}} \hat{u}+e_{y}\right)+e_{u}
\end{aligned}
$$

from which it follows that $\left(I-D_{\mathbf{C}} D_{\mathbf{P}}\right) \hat{u}=C_{\mathbf{C}} x_{c}+$ $D_{\mathbf{C}} C_{\mathbf{P}} x_{p}+D_{\mathbf{C}} e_{y}+e_{u}$. As such, we have that

$$
\begin{aligned}
\hat{u}=\left(I-D_{\mathbf{C}} D_{\mathbf{P}}\right)^{-1} & \cdot\left(C_{\mathbf{C}} x_{c}+D_{\mathbf{C}} C_{\mathbf{P}} x_{p}+D_{\mathbf{C}} e_{y}+e_{u}\right) .
\end{aligned}
$$

and similarly it can be obtained that

$$
\begin{aligned}
& \hat{y}=\left(I-D_{\mathbf{P}} D_{\mathbf{C}}\right)^{-1} \\
& \cdot\left(C_{\mathbf{p}} x_{p}+D_{\mathbf{P}} C_{\mathbf{C}} x_{c}+D_{\mathbf{P}} e_{u}+e_{y}\right)
\end{aligned}
$$

provided that the inverses in (10) exist. It is easy to see that $\left(I-D_{\mathbf{C}} D_{\mathbf{P}}\right)^{-1}$ as in (10a) exists if and only if $\left(I-D_{\mathbf{P}} D_{\mathbf{C}}\right)^{-1}$ as in (10b) does [28]. Hence, similar to the case in which no communication network is present, see, e.g., [31,35], to construct our hybrid model we need the following well-posedness assumption.

Standing Assumption 1 For the NCS described by (1)-(4) the interconnection is well-posed in the sense that $\left(I-D_{\mathbf{C}} D_{\mathbf{P}}\right)^{-1}$ (or, equivalently, $\left.\left(I-D_{\mathbf{P}} D_{\mathbf{C}}\right)^{-1}\right)$ exists.

Note that this well-posedness assumption is always satisfied when only the plant or the controller has feedthrough terms (in which case either $D_{\mathbf{P}}=0$ or $\left.D_{\mathbf{C}}=0\right)$, see also Remark 9 in Section 5. Moreover, based on Neumann series, see, e.g., [29], a sufficient condition for guaranteeing well-posedness of the interconnection is to require that

$$
\lim _{n \rightarrow \infty}\left(D_{\mathbf{C}} D_{\mathbf{P}}\right)^{n}=0,
$$

which is the case when the matrix $D_{\mathbf{C}} D_{\mathbf{P}}$ is Schur, i.e.,

$$
\max _{k}\left|\lambda_{k}\left(D_{\mathbf{C}} D_{\mathbf{P}}\right)\right|<1,
$$

where $\lambda_{k}$ denotes the $k$-th eigenvalue. Observe that the condition (11) can be related to a small-gain type of condition for feedback systems between the $\hat{u}$ - and $\hat{y}$-systems in (10). Indeed, since the interconnection between the $\hat{u}$ - and $\hat{y}$-systems in (10) is directly related to the system matrices $D_{\mathbf{P}}$ and $D_{\mathbf{C}}$, condition (11) imposes some sort of bound on the product of these matrices, just like small-gain theorems require that the product of given interconnection gains have to be strictly less than one.

By using (10) we can eliminate the control variables in the state dynamics. Moreover, by using the $\mathrm{ZOH}$ assumption in combination with (1), (2), and (7), the same can be done for the error dynamics, i.e., we have that

$$
\left(\dot{e}_{y}, \dot{e}_{u}\right)=\left(-C_{\mathbf{P}} \dot{x}_{p},-C_{\mathbf{C}} \dot{x}_{c}\right) .
$$

Combining the above and taking (3) into consideration, the triple $(\mathcal{P}, \mathcal{C}, \mathcal{N})$ can be rewritten into the hybrid 


$$
\begin{aligned}
& \mathbf{A}=\left[\begin{array}{cc}
A_{\mathbf{P}}+B_{\mathbf{P}}\left(I_{m_{u}}-D_{\mathbf{C}} D_{\mathbf{P}}\right)^{-1} D_{\mathbf{C}} C_{\mathbf{P}} & B_{\mathbf{P}}\left(I_{m_{u}}-D_{\mathbf{C}} D_{\mathbf{P}}\right)^{-1} C_{\mathbf{C}} \\
B_{\mathbf{C}}\left(I_{m_{y}}-D_{\mathbf{P}} D_{\mathbf{C}}\right)^{-1} C_{\mathbf{P}} & A_{\mathbf{C}}+B_{\mathbf{C}}\left(I_{m_{y}}-D_{\mathbf{P}} D_{\mathbf{C}}\right)^{-1} D_{\mathbf{P}} C_{\mathbf{C}}
\end{array}\right], \quad \mathbf{C}=\left[\begin{array}{cc}
-C_{\mathbf{P}} & 0 \\
0 & -C_{\mathbf{C}}
\end{array}\right] \mathbf{A}, \\
& \mathbf{E}=\left[\begin{array}{cc}
B_{\mathbf{P}}\left(I_{m_{u}}-D_{\mathbf{C}} D_{\mathbf{P}}\right)^{-1} D_{\mathbf{C}} & B_{\mathbf{P}}\left(I_{m_{u}}-D_{\mathbf{C}} D_{\mathbf{P}}\right)^{-1} \\
B_{\mathbf{C}}\left(I_{m_{y}}-D_{\mathbf{P}} D_{\mathbf{C}}\right)^{-1} & B_{\mathbf{C}}\left(I_{m_{y}}-D_{\mathbf{P}} D_{\mathbf{C}}\right)^{-1} D_{\mathbf{P}}
\end{array}\right], \quad \quad \mathbf{F}=\left[\begin{array}{cc}
-C_{\mathbf{P}} & 0 \\
0 & -C_{\mathbf{C}}
\end{array}\right] \mathbf{E} .
\end{aligned}
$$

system formalism advocated in [23]. To do so, similar to $[5,7,12,14,15,23,30]$, we introduce the timer $\tau \in$ $\mathbb{R}_{\geq 0}$, which keeps track of the time elapsed since the last transmission and resets to zero after a transmission has occurred, and the counter $\kappa \in \mathbb{N}$, which keeps track of the number of transmissions. Using these auxiliary variables, the NCS can be expressed as the hybrid model ${ }^{1}$

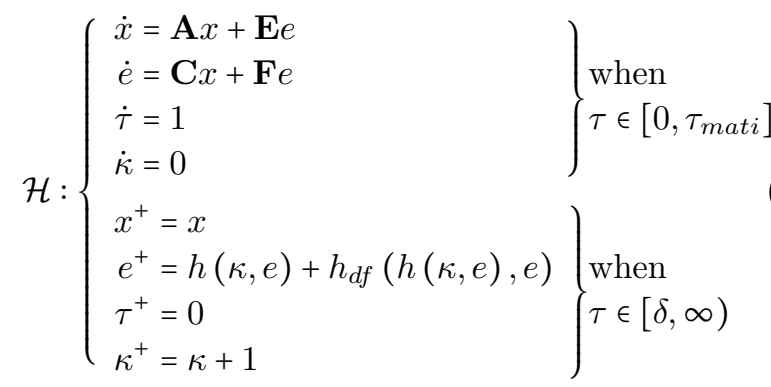

where $x:=\left(x_{p}, x_{c}\right) \in \mathbb{R}^{m_{x}}$, the matrices $\mathbf{A}, \mathbf{C}, \mathbf{E}, \mathbf{F}$ are given by (13), and with the full state of the hybrid system

$$
\xi:=(x, e, \tau, \kappa) \in \mathbb{X}:=\mathbb{R}^{m_{x}} \times \mathbb{R}^{m_{e}} \times \mathbb{R}_{\geq 0} \times \mathbb{N} .
$$

Using this hybrid modeling framework, stability in the sense of UGES for the NCS will be analyzed.

Remark 2 It is also possible to perform an $\mathcal{L}_{2}$-induced gain analysis for the NCS if the plant $\mathcal{P}$ is also modeled with an external (disturbance) input and (performance) output. However, for the sake of readability and brevity, in this paper we limit ourselves to the analysis of UGES.

\section{$3 \quad$ Stability analysis}

In this section, we analyze the stability of the hybrid model (12) for the NCS. Hereto, consider the following.

Definition 3 For the overall (hybrid) system $\mathcal{H}$ given by (12) with (14), the set

$$
\mathcal{E}=\{\xi \in \mathbb{X} \mid x=0 \wedge e=0\}
$$

is said to be uniformly globally exponentially stable (UGES) if there exists a function $\beta: \mathbb{R}_{\geq 0} \times \mathbb{R}_{\geq 0} \times \mathbb{N} \rightarrow \mathbb{R}_{\geq 0}$ of the form $\beta(r, t, j)=M r \exp (-\rho(t+j))$ for some $M>0$ and $\rho>0$, such that for any initial condition $\xi(0,0) \in \mathbb{X}$, all corresponding maximal solutions $\xi$ are complete and satisfy for all $(t, j) \in \operatorname{dom} \xi$

$$
|(x(t, j), e(t, j))| \leq \beta(|(x(0,0), e(0,0))|, t, j) .
$$

\footnotetext{
1 For more details on hybrid systems of the form of (12) the interested reader is referred to [11].
}

Remark 4 In Definition 3 we used the solution concept and terminology for hybrid systems as introduced in [11] for describing the NCS in terms of the hybrid system (12). For more details on solutions, completeness, and maximality the interested reader is referred to [5, 11, 14, 23].

Based now on the results in [5, 23], we provide LMIbased conditions that guarantee UGES of the set (15). However, to do so, the way of viewing the scheduling protocol first needs to be re-examined.

\subsection{UGES scheduling protocols}

One of the most important aspects in the analysis approach of [23] is the notion of UGES scheduling protocols, which we need to modify to include the presence of direct-feedthrough terms. In particular, we need to take into account that in this paper, as a result of the presence of the update function $h_{d f}$ in (9), the update of the error $e$ depends on the system matrices $D_{\mathbf{P}}$ and $D_{\mathbf{C}}$, while in [23] (9) solely depends on the scheduling law and not on the controller/plant parameters (i.e., $h_{d f}=0$ in [23]). To this end, the update equation of the error is modeled as a discrete-time system

$$
e(i+1)=p_{f}(i, e(i))
$$

induced by a certain function $p_{f}: \mathbb{N} \times \mathbb{R}^{m_{e}} \rightarrow \mathbb{R}^{m_{e}}$, which, according to (9), is given for all $i \in \mathbb{N}$ and $e \in \mathbb{R}^{m_{e}}$ by

$$
p_{f}(i, e)=h(i, e)+h_{d f}(i, e) .
$$

Consider now the following definition.

Definition 5 Let $D_{\mathbf{P}} \in \mathbb{R}^{m_{y} \times m_{u}}$ and $D_{\mathbf{C}} \in \mathbb{R}^{m_{u} \times m_{y}}$ be given. The scheduling protocol (function) $h: \mathbb{N} \times \mathbb{R}^{m_{e}} \rightarrow$ $\mathbb{R}^{m_{e}}$ is said to be $\left(D_{\mathbf{P}}, D_{\mathbf{C}}\right)$-UGES if the discrete-time system (16) with (17) is UGES, or, in other words, admits a (Lyapunov) function $W: \mathbb{N} \times \mathbb{R}^{m_{e}} \rightarrow \mathbb{R}_{\geq 0}$, and constants $\lambda \in[0,1)$ and $\underline{\alpha}_{W}^{c}, \bar{\alpha}_{W}^{c}>0$ such that for all $i \in \mathbb{N}$ and all $e \in \mathbb{R}^{m_{e}}$ it holds that

$$
\begin{aligned}
\underline{\alpha}_{W}^{c}|e| \leq W(i, e) & \leq \bar{\alpha}_{W}^{c}|e| \\
W\left(i+1, p_{f}(i, e)\right) & \leq \lambda W(i, e) .
\end{aligned}
$$

Sometimes we also say in this case that the scheduling protocol is $\left(D_{\mathbf{P}}, D_{\mathbf{C}}\right)$-UGES with Lyapunov function $W$.

Definition 5 describes a generalization of the concept of UGES scheduling protocols as introduced in [23]. In particular, when Definition 5 is satisfied by the discretetime system (16) with (17) where $D_{\mathbf{P}}=0$ and $D_{\mathbf{C}}=0$ (and thus $h_{d f}=0$ ), as was the case in [23], we recover the definition of UGES scheduling protocols (i.e., the protocol function $h$ is UGES with Lyapunov function 
$W)$. Hence, the notion of UGES scheduling protocols from [23] is equal to $(0,0)$-UGES scheduling protocols. As shown in [23], various scheduling protocols exist that satisfy this definition of $(0,0)$-UGES scheduling protocols, including the SD, RR, and TOD protocols.

In Section 4 , we analyze the concept of $\left(D_{\mathbf{P}}, D_{\mathbf{C}}\right)$-UGES scheduling protocols in more detail. Moreover, in Section 5, we will show that various well-known scheduling protocols from $[23,30]$ are also $\left(D_{\mathbf{P}}, D_{\mathbf{C}}\right)$-UGES according to Definition 5 under appropriate conditions on the direct-feedthrough matrices $D_{\mathbf{P}}$ and $D_{\mathbf{C}}$.

\subsection{LMI-based condition for UGES}

We now recapitulate the main results of $[5,23]$ concerning UGES for the NCS given by (1)-(4) and modeled by (12), while also taking Definition 5 into account. In particular, we formulate LMI-based conditions guaranteeing the set $\mathcal{E}$ in (15) to be UGES for the NCS with direct-feedthrough terms modeled by (12).

Theorem 6 Consider the system $\mathcal{H}$ of (12) that satisfies Assumption 1. Assume there exist a function $W: \mathbb{N} \times$ $\mathbb{R}^{m_{e}} \rightarrow \mathbb{R}_{\geq 0}$, a symmetric positive definite matrix $X_{\mathbf{T}}$, and strictly positive real numbers $M, \underline{\alpha}_{W}^{c}, \bar{\alpha}_{W}^{c}, \lambda \in(0,1)$, and $0<\varepsilon<\gamma$, and suppose that the following holds:

(1) The scheduling protocol (function) $h: \mathbb{N} \times \mathbb{R}^{m_{e}} \rightarrow$ $\mathbb{R}^{m_{e}}$ is $\left(D_{\mathbf{P}}, D_{\mathbf{C}}\right)$-UGES with Lyapunov function $W$ and the constants $\underline{\alpha}_{W}^{c}, \bar{\alpha}_{W}^{c}$, and $\lambda$.

(2) For all $\kappa \in \mathbb{N}$, and for almost all $\in \in \mathbb{R}^{m_{e}}$ it holds that

$$
\left|\frac{\partial W(\kappa, e)}{\partial e}\right| \leq M
$$

(3)

$\left[\begin{array}{cc}\mathbf{A}^{\top} X_{\mathbf{T}}+X_{\mathbf{T}} \mathbf{A}+\varepsilon^{2} I_{m_{x}}+M^{2} \mathbf{C}^{\top} \mathbf{C} & X_{\mathbf{T}} \mathbf{E} \\ \mathbf{E}^{\top} X_{\mathbf{T}} & -\underline{\alpha}_{W}^{c}{ }^{2}\left[\gamma^{2}-\varepsilon^{2}\right] I_{m_{e}}\end{array}\right] \leq 0$.

(4) $\tau_{\text {mati }}$ satisfies the bound

$$
\begin{aligned}
& \tau_{\text {mati }} \leq \begin{cases}\frac{1}{L r} \arctan \left(\frac{r(1-\lambda)}{2 \frac{\lambda}{1+\lambda}\left(\frac{\gamma}{L}-1\right)+1+\lambda}\right) & \gamma>L \\
\frac{1}{L} \frac{1-\lambda}{1+\lambda} & \gamma=L \\
\frac{1}{L r} \operatorname{arctanh}\left(\frac{r(1-\lambda)}{2 \frac{\lambda}{1+\lambda}\left(\frac{\gamma}{L}-1\right)+1+\lambda}\right) & \gamma<L \\
\frac{1}{\gamma} \arctan \left(\frac{(1+\lambda)(1-\lambda)}{2 \lambda}\right) & L=0\end{cases} \\
& \text { with } r=\sqrt{\left|\left(\frac{\gamma}{L}\right)^{2}-1\right| \text { and } L=M\left(\underline{\alpha}_{W}^{c}\right)^{-1}|\mathbf{F}| .}
\end{aligned}
$$

Then, the set $\mathcal{E}$ in (15) is UGES.

Note that Theorem 6 is merely an application of [5, Theorem 1]; its proof is therefore omitted, however, for more details see, e.g., [15] or [17]. Since $\gamma$ is the only free variable for the computation of the bound for $\tau_{m a t i}$ as $\lambda$ follows from the scheduling protocol, $\tau_{\text {mati }}$ can be maximized by means of minimizing $\gamma$ subject to $0<\varepsilon<\gamma$ and the LMI (20).
In order to use Theorem 6 , and, in particular, the LMI (20), to verify stability of the hybrid system (12), it is necessary to have a scheduling protocol function $h$ that is $\left(D_{\mathbf{P}}, D_{\mathbf{C}}\right)$-UGES such that (18) and (19) are satisfied.

\section{$4\left(D_{\mathbf{P}}, D_{\mathbf{C}}\right)$-UGES scheduling protocols}

To study if a scheduling protocol is $\left(D_{\mathbf{P}}, D_{\mathbf{C}}\right)$-UGES, we consider the update equation of the error to be modeled as the discrete-time system given by

$$
\begin{aligned}
e(i+1)=h & (i, e(i)) \\
& +\underbrace{\left[\begin{array}{ll}
0_{m_{y}} & D_{\mathbf{P}} \\
D_{\mathbf{C}} & 0_{m_{u}}
\end{array}\right]}_{=: \mathbf{D}}(e(i)-h(i, e(i))),
\end{aligned}
$$

see $(9),(16)$, and (17). In this section, we will provide conditions on the direct-feedthrough matrices $D_{\mathbf{P}}$ and $D_{\mathbf{C}}$ such that $((0,0)-)$ UGES scheduling protocols are also $\left(D_{\mathbf{P}}, D_{\mathbf{C}}\right)$-UGES scheduling protocols.

To this end, consider the situation in which $\ell \in \mathbb{N}_{>0}$ nodes are competing for access to the communication network, and, hence, the error vector can now be partitioned as $e(i)=\left(e_{1}(i), e_{2}(i), \ldots, e_{\ell}(i)\right)$, after reordering if necessary. As such, we can in general model the considered scheduling protocol functions as

$$
h(i, e)=\left(I_{m_{e}}-\Psi(i, e)\right) e
$$

for all $i \in \mathbb{N}$ and $e \in \mathbb{R}^{m_{e}}$ with $\Psi(i, e)=\operatorname{diag}\left\{\psi_{1}(i, e) I_{m_{1}}\right.$, $\left.\psi_{2}(i, e) I_{m_{2}}, \ldots, \psi_{\ell}(i, e) I_{m_{\ell}}\right\}$, where $I_{m_{k}}$ are identity matrices with $m_{k}$ the dimension of the $k$-th node in the network such that $\sum_{k=1}^{\ell} m_{k}=m_{e}$ and where $\psi_{k}(i, e)$ equals one when the $k$-th node is updated and is zero when it is not. Many well-known scheduling protocols, such as the SD, RR, and TOD protocols can be modeled according to (23), see Section 5 or [23].

Based on (23), we can decompose the system (22) as

$$
\begin{aligned}
e(i+1) & =h(i, e(i))+\mathbf{D} w(i) \\
w(i) & =\Psi(i, e(i)) e(i),
\end{aligned}
$$

from which we can state the following theorem regarding UGES of the scheduling protocols modeled by (23).

Theorem 7 Let $D_{\mathbf{P}} \in \mathbb{R}^{m_{y} \times m_{u}}$ and $D_{\mathbf{C}} \in \mathbb{R}^{m_{u} \times m_{y}}$ be given. Assume there exists a function $W: \mathbb{N} \times \mathbb{R}^{m_{e}} \rightarrow \mathbb{R}_{\geq 0}$ that is globally Lipschitz in its second argument with Lipschitz constant $M>0$, i.e., satisfying (19), and suppose that the following holds:

(1) The scheduling protocol (function) $h: \mathbb{N} \times \mathbb{R}^{m_{e}} \rightarrow$ $\mathbb{R}^{m_{e}}$ modeled by (23) is (0,0)-UGES with Lyapunov function $W$, i.e., there exist constants $\lambda \in[0,1)$ and $\underline{\alpha}_{W}^{c}, \bar{\alpha}_{W}^{c}>0$ such that for all $i \in \mathbb{N}$ and $e \in \mathbb{R}^{m_{e}}$ (18) holds with (17) and $h_{d f}=0$. 
(2) For the matrix $\mathbf{D}$ defined in (22), it holds that

$$
|\mathbf{D}|<\frac{\underline{\alpha}_{W}^{c}}{M}(1-\lambda) \text {. }
$$

Then, the scheduling protocol (function) $h$ is $\left(D_{\mathbf{P}}, D_{\mathbf{C}}\right)$ UGES with Lyapunov function $W$, where (18a) is satisfied with $\underline{\alpha}_{W}^{c}$ and $\bar{\alpha}_{W}^{c}$ and $(18 \mathrm{~b})$ with the decreasing rate $\rho:=\lambda+\left(\underline{\alpha}_{W}^{c}\right)^{-1} M|\mathbf{D}| \in[0,1)$.

The proof is given in Appendix A. Observe now that the first item in Theorem 7 covers the class of UGES scheduling protocols as introduced in [23]. As such, Theorem 7 states that any scheduling protocol that is UGES in the classical sense of $[23$, Definition 7$]$ is also a $\left(D_{\mathbf{P}}, D_{\mathbf{C}}\right)$ UGES scheduling protocol when (24) is satisfied, provided it admits a globally Lipschitz Lyapunov function, which is often the case, see Section V in [23]. Theorem 7 also confirms what one would intuitively expect, i.e., that when $h$ is a UGES scheduling protocol as introduced in [23] and the direct-feedthrough terms are "small enough", the discrete-time system given by (22) is also UGES. For the well-known SD, RR, and TOD protocols, see $[5,23,30]$, the required bounds on the norm of the matrix $\mathbf{D}$ are summarized in Table 1, where we recall that the number of nodes is denoted by $\ell \in \mathbb{N}_{>0}$.

Table 1

UGES scheduling protocol condition for the SD, RR, and TOD protocol, where $\ell$ is the number of nodes.

\begin{tabular}{|c|c|c|c|l|}
\hline Protocol & $\underline{\alpha}_{W}^{c}$ & $M$ & $\lambda$ & $|\mathbf{D}|<c$, where \\
\hline SD & 1 & 1 & 0 & $c=1$ \\
RR & 1 & $\sqrt{\ell}$ & $\sqrt{\frac{\ell-1}{\ell}}$ & $c=\frac{\sqrt{\ell}-\sqrt{\ell-1}}{\ell}$ \\
TOD & 1 & 1 & $\sqrt{\frac{\ell-1}{\ell}}$ & $c=1-\sqrt{\frac{\ell-1}{\ell}}$ \\
\hline
\end{tabular}

From these results one can directly see that there exists a relation between the number of nodes $\ell$ and the "size" of the direct-feedthrough terms, i.e., when the number of nodes $\ell$ increases, the stricter the condition on the matrix $\mathbf{D}$ becomes (to apply Theorems 6 and 7 to obtain UGES of the NCS). One can also observe that, in general, the TOD protocol requires a less strict condition than the RR protocol. This is already a first indication that for certain NCSs with direct-feedthrough terms the corresponding discrete-time system (16) with (17) might be stable with one UGES protocol, but not with another, which is a large difference with the results obtained in [23]. In fact, this observation will be justified in the following sections.

While Theorem 7 in general can be applied to any NCS with direct-feedthrough terms for any UGES scheduling protocol function $h$ that admits a globally Lipschitz Lyapunov function, the obtained condition on the matrix $\mathbf{D}$ is subject to some conservatism. Fortunately, it can be shown that for some protocols we can obtain less conservative results by exploiting the specific structure of these protocol functions. This is the topic of the next section.

\section{$5 \quad\left(D_{\mathbf{P}}, D_{\mathbf{C}}\right)$-UGES of specific protocols}

In this section, we focus on some of the most wellknown scheduling protocols, i.e., the SD, RR, and TOD protocols, and show that the conditions on the directfeedthrough terms under which those protocols are $\left(D_{\mathbf{P}}, D_{\mathbf{C}}\right)$-UGES, see Table 1, can be relaxed.

\subsection{Sampled-data protocol}

First we consider the sampled-data (SD) protocol, see, e.g., $[23,30]$, that is modeled for $i \in \mathbb{N}$ and $e \in \mathbb{R}^{m_{e}}$ by

$$
h(i, e)=0,
$$

which is indeed of the form (23), implying that an update of the error according to (22) is now given by

$$
e(i+1)=\mathbf{D} e(i) .
$$

Hence, the SD protocol is $\left(D_{\mathbf{P}}, D_{\mathbf{C}}\right)$-UGES if and only if the matrix $\mathbf{D}$ is Schur, i.e., for all eigenvalues $\lambda_{k}$ of $\mathbf{D}$ it holds that

$$
\left|\lambda_{k}(\mathbf{D})\right|<1
$$

see, e.g., [19]. Note now that condition (26) is equivalent to the sufficient well-posedness condition of (11). As such, we have the following result for the SD protocol.

Proposition 8 Let $D_{\mathbf{P}} \in \mathbb{R}^{m_{y} \times m_{u}}$ and $D_{\mathbf{C}} \in \mathbb{R}^{m_{u} \times m_{y}}$ be given. The $S D$ protocol, modeled by the scheduling protocol function (25), is $\left(D_{\mathbf{P}}, D_{\mathbf{C}}\right)$-UGES if and only if (11) (cf. (26)) holds. Moreover, when this is the case, a Lyapunov function can be obtained as $W(e)=\sqrt{e^{\top} P e}$, where the real symmetric matrix $P>0$ is computed by solving

$$
\mathbf{D}^{\top} P \mathbf{D}-\rho P \leq 0
$$

for a certain constant $\rho \in[0,1)$, implying that (18a) is satisfied with $\underline{\alpha}_{W}^{c}=\sqrt{\lambda_{\min }(P)}$ and $\bar{\alpha}_{W}^{c}=\sqrt{\lambda_{\max }(P)}$, (18b) with $\lambda=\sqrt{\rho}$, and (19) with $M=\sqrt{\lambda_{\max }(P)}$.

Proposition 8 implies that, for any NCS given by (1)-(4) satisfying the sufficient well-posedness condition of (11), a solution to the LMI (27) exists, and, hence, Theorem 6 can always be used to obtain a bound on the MATI and thus guarantee UGES of the set $\mathcal{E}$ in (15) for the linear NCS given by (1)-(4) with the SD protocol as the scheduling protocol. Observe also that, based on (21), the "freedom" in (27) can be used for the optimization of the MATI.

Remark 9 The condition (26) (cf. (11)) implies that, when either $D_{\mathbf{P}}=0$ or $D_{\mathbf{C}}=0$ and, hence, $D_{\mathbf{C}} D_{\mathbf{P}}=0$, the other-ones matrix norm $\left(D_{\mathbf{P}} \neq 0\right.$ or $\left.D_{\mathbf{C}} \neq 0\right)$ can grow arbitrarily large. As a result, the $S D$ protocol is $\left(D_{\mathbf{P}}, 0\right)$ - and $\left(0, D_{\mathbf{C}}\right)$-UGES for any $D_{\mathbf{P}} \in \mathbb{R}^{m_{y} \times m_{u}}$ and $D_{\mathbf{C}} \in \mathbb{R}^{m_{u} \times m_{y}}$.

Remark 10 When we have that $|\mathbf{D}|<1$, the matrix $P$ in (27) can be taken as the identity matrix. As such, 
we have that $W(e)=|e|$ as proposed in [23] for the $S D$ protocol, and, hence, we recover the condition (24) for the SD protocol as stated in Theorem 7 (see Table 1). In this case, (18) and (19) are satisfied for $\underline{\alpha}_{W}^{c}=\bar{\alpha}_{W}^{c}=M=1$, and $\lambda=|\mathbf{D}|<1$.

\subsection{Round-robin protocol}

Secondly, we consider the round-robin (RR) protocol, which is also of the form (23), where in addition the value of the matrix $\Psi(i, e)$, which we write $\Delta(i)$ in this section, is only determined by the discrete-time $i$. More precisely, the RR protocol can be modeled according to

$$
h(i, e)=\left(I_{m_{e}}-\Delta(i)\right) e,
$$

for $i \in \mathbb{N}$ and $e \in \mathbb{R}^{m_{e}}$ where $\Delta(i)=\operatorname{diag}\left\{\Delta_{1}(i), \Delta_{2}(i), \ldots\right.$, $\left.\Delta_{\ell}(i)\right\}$ with the square matrices $\Delta_{k}(i)=\delta_{k}(i) I_{m_{k}}$ of dimension $m_{k}$, where

$$
\delta_{k}(i)= \begin{cases}1, & \text { if } i=k+j \ell, \text { for some } j \in \mathbb{N} \\ 0, & \text { otherwise }\end{cases}
$$

Let now $\ell_{y} \in \mathbb{N}_{>0}$ denote the number of nodes which are not associated to any actuator and, similarly, let $\ell_{u} \in \mathbb{N}_{>0}$ denote the number of nodes which are not associated to any sensor (note that $\ell_{y}+\ell_{u} \leq \ell$ ). As the scheduling rule of the RR protocol is a priori known, we can compute the trajectory for the discrete-time system given by $(22)$ with (28). As such, in the case that there are no mixed communication nodes present in the NCS, i.e., $\ell=\ell_{y}+$ $\ell_{u}$ and $e(i)=\left(e_{1}(i), \ldots, e_{\ell_{y}}(i), e_{\ell_{y}+1}(i), \ldots, e_{\ell_{y}+\ell_{u}}(i)\right)=$ $\left(e_{y}(i), e_{u}(i)\right)$ (after reordering the error, if needed), it can be shown that the evolution of the discrete-time system given by $(22)$ after $j \ell$ steps, $j \in \mathbb{N}$, is given by (see also Appendix B)

$$
\begin{aligned}
& e(j \ell)= \\
& {\left[\begin{array}{l}
e_{y}(j \ell) \\
e_{u}(j \ell)
\end{array}\right]=\left[\begin{array}{c}
\left(D_{\mathbf{P}} D_{\mathbf{C}}\right)^{j-1} D_{\mathbf{P}} e_{u}(0)+\left(D_{\mathbf{P}} D_{\mathbf{C}}\right)^{j} e_{y}(0) \\
\mathbf{0}_{m_{u}}
\end{array}\right] .}
\end{aligned}
$$

This implies that, when $j \rightarrow \infty$, we have an infinite repetition of either the terms $D_{\mathbf{P}} D_{\mathbf{C}}$ or $D_{\mathbf{C}} D_{\mathbf{P}}$ in the error $e_{y}$, while $e_{u}$ is always zero after $j \ell$ time steps. Hence, when (11) holds, i.e., when the matrix D is Schur, we have convergence of the error to zero, i.e.,

$$
\lim _{i \rightarrow \infty} e(i) \rightarrow 0 \text {. }
$$

In fact, from (30) if follows that

$$
|e(M)| \leq\left|\left[\begin{array}{cc}
D_{\mathbf{P}} & D_{\mathbf{P}} D_{\mathbf{C}} \\
0 & 0
\end{array}\right]\right|\left|D_{\mathbf{P}} D_{\mathbf{C}}\right|^{M}|e(0)|
$$

for sufficiently large $M \in \mathbb{N}$, implying that there exists a finite-step Lyapunov function, see, e.g., [10], showing UGES of the discrete-time system (22) modeled with the RR protocol given by $(28)$. Hence, $\left(D_{\mathbf{P}}, D_{\mathbf{C}}\right)$-UGES of the RR protocol is clearly guaranteed for the case of no mixed nodes when the sufficient condition of (11) for well-posedness is satisfied. As such, we can compose the following result for the RR protocol (the proof is given in Appendix A).

Proposition 11 Let $D_{\mathbf{P}} \in \mathbb{R}^{m_{y} \times m_{u}}$ and $D_{\mathbf{C}} \in \mathbb{R}^{m_{u} \times m_{y}}$ be given. The RR protocol, modeled by the scheduling protocol function of (28) with (29) and where $l=l_{u}+l_{y}$, is $\left(D_{\mathbf{P}}, D_{\mathbf{C}}\right)-U G E S$ if and only if (11) holds. Moreover, when this is the case, a Lyapunov function can be obtained as $W(e(i))=\sqrt{e^{\top}(i) P(i) e(i)}$, where the $\ell$-periodic matrices $P(k)=P(k+\ell)$ with $P(k)>0$ are computed by solving

$$
A_{k}^{\top} P(k+1) A_{k}-\rho(k) P(k) \leq 0
$$

for certain $\ell$-periodic scalars $\rho(k)=\rho(k+\ell) \in[0,1)$ for all $k \in \bar{\ell}:=\{1,2, \ldots, \ell\}$ and where the matrices $A_{k}$ are given by

$$
\begin{aligned}
& A_{k}:=\operatorname{diag}\left\{I_{m_{1}}, \ldots, I_{m_{k-1}}, 0_{m_{k}}, I_{m_{k+1}}, \ldots, I_{m_{\ell}}\right\} \\
& \quad+\mathbf{D} \operatorname{diag}\left\{0_{m_{1}}, \ldots, 0_{m_{k-1}}, I_{m_{k}}, 0_{m_{k+1}}, \ldots, 0_{m_{\ell}}\right\},
\end{aligned}
$$

implying that (18a) is satisfied with

$\underline{\alpha}_{W}^{c}=\min _{k} \sqrt{\lambda_{\min }(P(k))}$ and $\bar{\alpha}_{W}^{c}=\max _{k} \sqrt{\lambda_{\max }(P(k))}$, (18b) is satisfied with $\lambda=\max _{k} \sqrt{\rho(k)}$, and (19) with $M=\max _{k} \sqrt{\lambda_{\max }(P(k))}$.

Remark 12 Proposition 11 is based on the situation in which all the sensor nodes are visited before the actuator nodes. The proposition also holds in the case in which all actuator nodes are first visited. However, a priori, we could also alternate between sensor and actuator nodes or do have mixed nodes in the network (i.e., communication nodes that contain both actuator(s) and sensor(s)), resulting (in some cases) in the loss of the property of the repetition of the terms $D_{\mathbf{P}} D_{\mathbf{C}}$ or $D_{\mathbf{C}} D_{\mathbf{P}}$. Fortunately in these situations, (31) (which is a set of LMIs and therefore amenable for computational verification [4]) can be used as necessary and sufficient conditions for verifying $\left(D_{\mathbf{P}}, D_{\mathbf{C}}\right)-U G E S$ of the $R R$ protocol.

Remark 13 The $S D$ protocol is a special case of the $R R$ protocol. Indeed, for $\ell=1$ we have that in $(32) A_{k}=\mathbf{D}$ for $k=1$, implying that for this special case (31) indeed simplifies to $(27)$.

\subsection{Try-once-discard protocol}

Finally, we consider the try-once-discard (TOD) protocol as introduced in [30], which, in contrast to the SD and RR protocols, employs dynamic scheduling. In particular, the TOD protocol allocates network resources to the node with the greatest (weighted) error norm at a transmission time. As such, the TOD protocol is often considered to be a "smarter" scheduling protocol than, for instance, the RR protocol. This verbal description for the TOD protocol can also be converted into the model description of the form (23), where the rationale of the TOD protocol itself (as explained above) is modeled by 

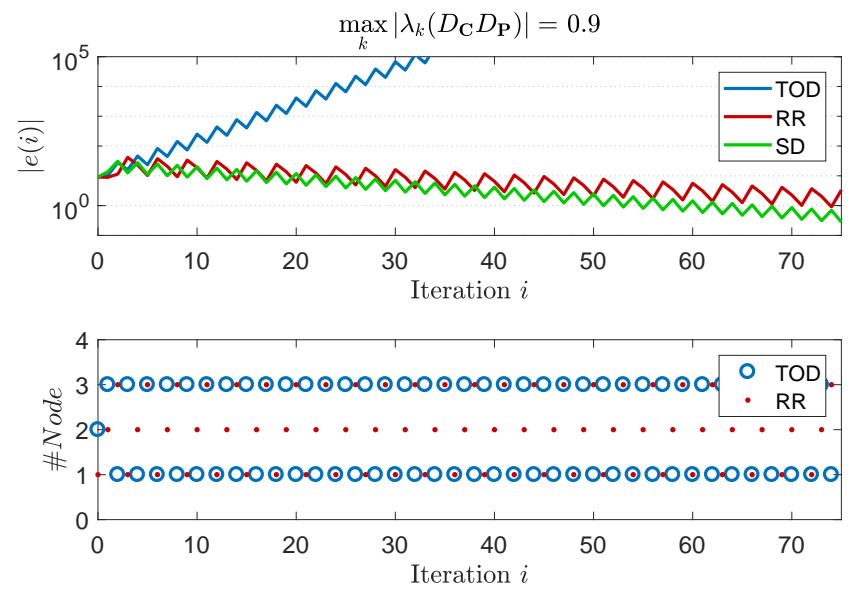

Fig. 2. The error evolution of the discrete-time system given by (22) satisfying (11) for the TOD, RR, and SD scheduling protocols and the indication for the TOD and RR protocols which node is updated at each time instant $i$.

$$
\psi_{k}(i, e)= \begin{cases}1, & \text { if } k=\min \left(\arg \max _{j}\left|e_{j}\right|\right) \\ 0, & \text { otherwise. }\end{cases}
$$

Based upon the sufficient conditions as presented in Table 1, one might expect, similar to the SD and RR protocols, that system (22) with the TOD protocol is also $\left(D_{\mathbf{P}}, D_{\mathbf{C}}\right)$-UGES when (11) is satisfied. This is because the TOD protocol has a less conservative bound in Table 1 compared to the RR protocol. However, a simple example shows that this is in general not true. Consider for instance system (22) with the number of nodes $\ell=3$, $\ell_{y}=2$ and $\ell_{u}=1$ (hence, we thus have two sensor nodes, one actuator node, and no mixed nodes), and with the direct-feedthrough matrices given by

$$
D_{\mathbf{P}}=\left[\begin{array}{c}
-3.5 \\
1.0
\end{array}\right] \text { and } D_{\mathbf{C}}=\left[\begin{array}{ll}
0.5 & 0.85
\end{array}\right] \text {. }
$$

As such, we can partition the error vector as $e=$ $\left(e_{y}, e_{u}\right)=\left(e_{y}^{1}, e_{y}^{2}, e_{u}\right)$. It is obvious that condition (11) is satisfied for the considered system as

$$
\max _{k}\left|\lambda_{k}\left(D_{\mathbf{C}} D_{\mathbf{P}}\right)\right|=0.9
$$

however, when we simulate the discrete-time system of (22) with (34) for the various protocols, we can see that exploiting the TOD protocol results in an unstable discrete-time system, see Fig. 2.

This example shows that, although the SD and RR protocols are $\left(D_{\mathbf{P}}, D_{\mathbf{C}}\right)$-UGES, the TOD protocol is not a $\left(D_{\mathbf{P}}, D_{\mathbf{C}}\right)$-UGES scheduling protocol even though (11) is satisfied. As such, the rationale of the TOD protocol is in general no longer appropriate when directfeedthrough terms are present in the system. In fact, as seen in the example, we can even have that exploiting the "smarter" TOD protocol is not always better than exploiting the RR protocol in the presence of directfeedthrough terms.
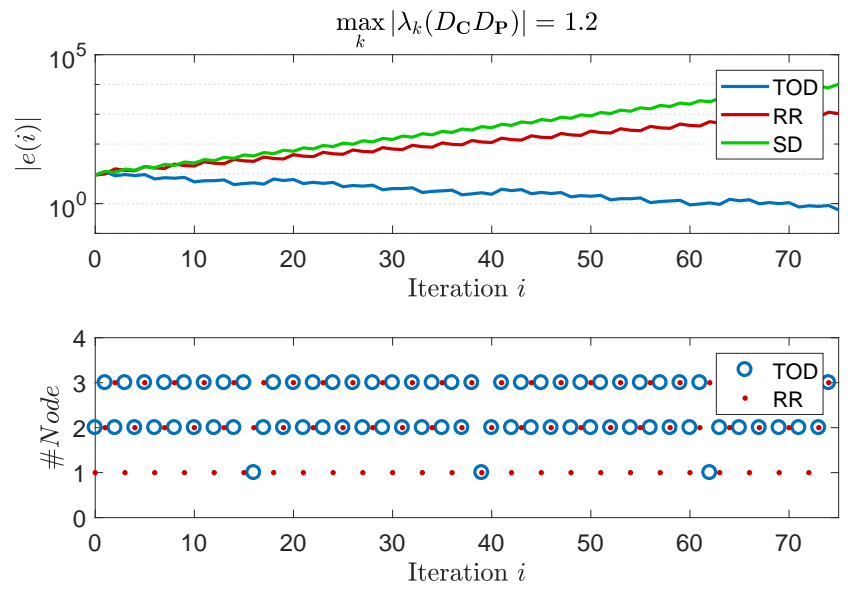

Fig. 3. The error evolution of the discrete-time system given by (22) not satisfying (11) for the TOD, RR, and SD scheduling protocols and the indication for the TOD and RR protocols which node is updated at each time instant $i$.

On the other hand, it can also be shown that the TOD protocol may outperform both the RR and SD protocols. Consider hereto the same example, however now with

$$
D_{\mathbf{P}}=\left[\begin{array}{l}
-0.4 \\
-0.8
\end{array}\right] \text { and } D_{\mathbf{C}}=\left[\begin{array}{ll}
0.8 & 1.1
\end{array}\right] .
$$

For these direct-feedthrough matrices Assumption 1 is still satisfied, however (11) is not as

$$
\max _{k}\left|\lambda_{k}\left(D_{\mathbf{C}} D_{\mathbf{P}}\right)\right|=1.2 \text {. }
$$

As a result, based on Propositions 8 and 11, the SD and RR protocols will not be $\left(D_{\mathbf{P}}, D_{\mathbf{C}}\right)$-UGES, however, as can be seen from Fig. 3, the TOD protocol is $\left(D_{\mathbf{P}}, D_{\mathbf{C}}\right)$ UGES in this case. This example shows that communicating more data (e.g., using the SD protocol instead of the TOD protocol) also is not always better.

These counterintuitive results show that obtaining necessary and sufficient conditions, as we have for the SD and RR protocols, is not an easy task for the TOD protocol. However, we can again exploit the structure of the TOD protocol to obtain sufficient conditions that are in general less strict than the condition as presented in Table 1. In particular, consider the following. Suppose that, without loss of generality,

$$
\left|e_{k}\right|=\max _{j \in \bar{\ell}}\left|e_{j}\right|
$$

for some $k \in \bar{\ell}$ with $\bar{\ell}:=\{1,2, \ldots, \ell\}$, or, stated differently,

$$
\left|e_{k}\right|^{2}-\left|e_{j}\right|^{2} \geq 0, \quad \text { for all } j \neq k \text {. }
$$

Hence, according to (23) with (33) we have that

$$
h(i, e)=\operatorname{diag}\left\{I_{m_{1}}, \ldots, I_{m_{k-1}}, 0_{m_{k}}, I_{m_{k+1}}, \ldots, I_{m_{\ell}}\right\} e .
$$

Based on (35), we also introduce for each $k \in \bar{\ell}$ the sets

$$
\mathcal{C}_{k}:=\left\{e \in \mathbb{R}^{m_{e}} \mid e^{\top} Q_{k j} e \geq 0, j \in \bar{\ell} \backslash\{k\}\right\}
$$


with the matrices

$$
\begin{aligned}
Q_{k j}:=\operatorname{diag}\{ & 0_{m_{1}}, \ldots, 0_{m_{k-1}}, I_{m_{k}}, 0_{m_{k+1}}, \\
& \left.\ldots, 0_{m_{j-1}},-I_{m_{j}}, 0_{m_{j+1}}, \ldots, 0_{m_{\ell}}\right\} .
\end{aligned}
$$

By combining the above, the discrete-time system of (22) with (23) and (33) can be described as

$$
\begin{aligned}
e(i+1) & =\left(I_{m_{e}}-\Psi(i, e(i))\right) e(i)+\mathbf{D} \Psi(i, e(i)) e(i) \\
& =A_{k} e(i)
\end{aligned}
$$

when $e(i) \in \mathcal{C}_{k}$ for some $k \in \bar{\ell}$, with the matrix $A_{k}$ defined by (32) and where the matrix $\mathbf{D}$ is as in (22). Note now that (37) describes a piecewise linear ( $P W L$ ) discretetime system, implying that its stability (and, therefore, $\left(D_{\mathbf{P}}, D_{\mathbf{C}}\right)$-UGES of the TOD protocol) can be determined by solving a set of LMIs, see, e.g., [4]. In fact, we have the following result for the TOD protocol (the proof is given in Appendix A).

Proposition 14 Let $D_{\mathbf{P}} \in \mathbb{R}^{m_{y} \times m_{u}}$ and $D_{\mathbf{C}} \in \mathbb{R}^{m_{u} \times m_{y}}$ be given. The TOD protocol, modeled by the scheduling protocol function of (23) with $(33)$, is $\left(D_{\mathbf{P}}, D_{\mathbf{C}}\right)-U G E S$ with Lyapunov function $W(e)=\sqrt{e^{\top} P e}$ if there exist a real symmetric matrix $P>0$, a constant $\rho \in(0,1)$, and nonnegative constants $\beta_{j}^{k}, k \in \bar{\ell}$ and $j \in \bar{\ell} \backslash\{k\}$, such that

$$
A_{k}^{\top} P A_{k}-\rho P+\sum_{j=1, j \neq k}^{\ell} \beta_{j}^{k} Q_{k j} \leq 0
$$

holds for all $k \in \bar{\ell}$ with the matrices $Q_{k j}$ given by (36) and where the matrix $A_{k}$ is given by (32). Moreover, (18a) is then satisfied for $\underline{\alpha}_{W}^{c}=\sqrt{\lambda_{\min }(P)}$ and $\bar{\alpha}_{W}^{c}=\sqrt{\lambda_{\max }(P)}$, (18b) for $\lambda=\sqrt{\rho}$, and (19) for $M=\sqrt{\lambda_{\max }(P)}$.

Remark 15 For the obtained class of PWL systems there is not one undisputed way of obtaining sufficient conditions such that UGES of the system given by (37) is guaranteed. As such, the sufficient conditions as proposed in Proposition 14, based on $S$-procedure relaxations as mentioned in the proof of Proposition 14 (see Appendix $A)$, are not the only way to go. For instance, an effective approach could also be to use versatile piecewise quadratic (PWQ) Lyapunov functions to obtain sufficient LMI conditions, see, e.g., [8,13]. However, due to space limitations, we restrict ourselves to the result of Proposition 14. For more information about stability of $P W L$ systems we refer to $[8,13,21]$ and the references therein.

Remark 16 The SD protocol is also a special case of the TOD protocol. Indeed, similar to Remark 13, we have that, for $\ell=1,(38)$ indeed simplifies to $(27)$.

\section{New scheduling protocols}

In the previous section we have seen that the rationale behind some (well-known) scheduling protocols can be a priori lost when direct-feedthrough terms are present in the NCS. Therefore, in this section, we propose some new $\left(D_{\mathbf{P}}, D_{\mathbf{C}}\right)$-UGES scheduling protocols, designed to work for "larger" values of $\left(D_{\mathbf{P}}, D_{\mathbf{C}}\right)$ and/or improve the convergence rate (i.e., smaller $\lambda$ ) of (22) and, hence, lead to larger values of the MATI via Theorem 6 .

\subsection{SD+ protocol}

The rationale behind the $\mathrm{SD}$ protocol is to update, at a given transmission time $t_{j}$, all networked values (of both the input $u$ as well as the output $y$ ) to their "true" values, see, e.g., [23,30], leading to the networked-induced error $e$ being completely reset to zero at $t_{j}$ (in absence of $D_{\mathbf{P}}$ and $\left.D_{\mathbf{C}}\right)$. However, from the SD protocol function given by (25), it can directly be observed that the overall networked-induced error $e$ is not completely reset to zero when either one of the matrices $D_{\mathbf{P}}$ or $D_{\mathbf{C}}$ is nonzero in (22). As such, we have that the rationale of the SD protocol is a priori lost when direct-feedthrough terms are present in the NCS.

Therefore, we introduce in this subsection an extended version of the SD protocol, for which we coin the term $\mathrm{SD}+$ protocol, modeled by

$$
h(i, e)=\left(\mathbf{D}-I_{m_{y}+m_{u}}\right)^{-1} \mathbf{D} e .
$$

Hence, provided that the inverse in (39) exists, which is the case in view of Standing Assumption 1, we now have that at a transmission time the error $e$ is always reset to zero, i.e., $e^{+}\left(t_{j}\right)=0$. As such, we can directly compose the following result for the $\mathrm{SD}+$ protocol.

Proposition 17 Let $D_{\mathbf{P}} \in \mathbb{R}^{m_{y} \times m_{u}}$ and $D_{\mathbf{C}} \in \mathbb{R}^{m_{u} \times m_{y}}$ be given. The $S D+$ protocol, modeled by the scheduling protocol function of $(39)$, is $\left(D_{\mathbf{P}}, D_{\mathbf{C}}\right)-U G E S$ if and only if Assumption 1 is satisfied. Moreover, when this is the case, a Lyapunov function is given by $W(e)=|e|$, implying that (18a) is satisfied for $\underline{\alpha}_{W}^{c}=\bar{\alpha}_{W}^{c}=1,(18 \mathrm{~b})$ for arbitrary small $\lambda \in[0,1)$, and (19) for $M=1$.

Observe that, as $\lambda$ can be taken arbitrarily small, the $\mathrm{SD}+$ protocol will always result in a higher value for the MATI than the SD protocol. In addition, where the SD protocol is limited to NCSs satisfying (11), the SD+ protocol guarantees stability for all NCSs satisfying Standing Assumption 1. As such, the SD+ protocol is a clear improvement upon the SD protocol.

Remark 18 When there are no direct feedthrough terms present in the overall system (i.e., $D_{\mathbf{P}}$ and $D_{\mathbf{C}}$ are zero), we recover $(25)$ again, making the $S D+$ protocol indeed an extension of the $S D$ protocol.

Remark 19 Based on the Schur complement and the Woodbury matrix identity to invert a matrix blockwise, see, e.g., [1,33], the scheduling protocol function of (39) can be rewritten as

$h(i, e)=\left[\begin{array}{cc}\left(D_{\mathbf{P}} D_{\mathbf{C}}-I\right)^{-1} D_{\mathbf{P}} D_{\mathbf{C}} & \left(D_{\mathbf{P}} D_{\mathbf{C}}-I\right)^{-1} D_{\mathbf{P}} \\ \left(D_{\mathbf{C}} D_{\mathbf{P}}-I\right)^{-1} D_{\mathbf{C}} & \left(D_{\mathbf{C}} D_{\mathbf{P}}-I\right)^{-1} D_{\mathbf{C}} D_{\mathbf{P}}\end{array}\right] e$, 
from which it follows that (39) corresponds to an update of the networked values according to

$$
\begin{aligned}
& \hat{y}^{+}=y+\left(D_{\mathbf{P}} D_{\mathbf{C}}-I_{m_{y}}\right)^{-1} D_{\mathbf{P}}\left(D_{\mathbf{C}} e_{y}+e_{u}\right) \\
& \hat{u}^{+}=u+\left(D_{\mathbf{C}} D_{\mathbf{P}}-I_{m_{u}}\right)^{-1} D_{\mathbf{C}}\left(D_{\mathbf{P}} e_{u}+e_{y}\right) .
\end{aligned}
$$

This implies that for the implementation of the $S D+$ protocol the sensor/actuator data needs to be available in a centralized manner, i.e., all nodes need to know about the values of all the other nodes or there is a need for a central coordinator. Fortunately, since the $S D+$ protocol updates all nodes at the same time, this is often not a problem.

\subsection{Switching scheduling protocol}

In many NCSs we have that the sensor and actuator nodes are separated, i.e., we have no mixed nodes in the NCS and thus $\ell_{y}+\ell_{u}=\ell$. As a result, from the structure of the discrete-time system (22), it is also possible to assume a separation of the errors $e_{y}$ and $e_{u}$ for these NCSs. That is, the update of the nodes corresponding to $e_{y} \in \mathbb{R}^{m_{y}}$ are now governed by

$$
h_{y}\left(i_{y}, e_{y}\right)=\left(I_{m_{y}}-\Psi_{y}\left(i_{y}, e_{y}\right)\right) e_{y}
$$

while we have that for the nodes corresponding to $e_{u} \epsilon$ $\mathbb{R}^{m_{u}}$ their update is modeled through

$$
h_{u}\left(i_{u}, e_{u}\right)=\left(I_{m_{u}}-\Psi_{u}\left(i_{u}, e_{u}\right)\right) e_{u},
$$

for all $i_{y}, i_{u} \in \mathbb{N}$ and where $\Psi_{y}$ and $\Psi_{u}$ are similar matrices as $\Psi$ in (23). Observe now that we also consider here a separation of the discrete-time $i$ in (22) by means of the introduction of the (discrete-) times $i_{y}$ and $i_{u}$, which act as "counters" that keep track of the number of sensor and actuator updates that have occurred, respectively, i.e., we have that $i=i_{y}+i_{u}$. Moreover, in the case that a sensor node is updated at time $i$, we have that the "counter" $i_{y}$ is incremented by one (and thus $i$ too). As such, in this case, we denote the next discrete time-step by $i+1=\left(i_{y}+1\right)+i_{u}$. Similarly, in the case that an actuator node is updated, we have that $i_{u}$ is incremented by one (and thus $i$ too), for which we denote the next discrete time-step by $i+1=i_{y}+\left(i_{u}+1\right)$.

Now, based on the separation of the errors, we propose to introduce the new switching scheduling protocol that uses a switching law $q: \mathbb{N} \times \mathbb{N} \times \mathbb{R}^{m_{e}} \rightarrow\{1,2\}$ that determines if either (one of) the sensor nodes are allowed to update according to $(40)$ (when $q\left(i_{y}, i_{u}, e\right)=1$ ) or (one of) the actuator nodes according to (41) (when $\left.q\left(i_{y}, i_{u}, e\right)=2\right)$. To be more precise, we have the following two situations:

- All the sensors nodes corresponding to the output $y$ can compete to access the channel to update. This corresponds to the error update according to

$$
\begin{aligned}
& e_{y}(i+1)=\left(I_{m_{y}}-\Psi_{y}\left(i_{y}, e_{y}(i)\right)\right) e_{y}(i) \\
& e_{u}(i+1)=e_{u}(i)+D_{\mathbf{C}} \Psi_{y}\left(i_{y}, e_{y}(i)\right) e_{y}(i)
\end{aligned}
$$

when $q\left(i_{y}, i_{u}, e\right)=1$ and where $i+1=\left(i_{y}+1\right)+i_{u}$ (i.e., the counter $i_{y}$ is incremented by one and thus $i$ too).
- All the actuator nodes corresponding to the input $u$ can compete to access the channel to update. This corresponds to the error update according to

$$
\begin{aligned}
& e_{y}(i+1)=e_{y}(i)+D_{\mathbf{P}} \Psi_{u}\left(i_{u}, e_{u}(i)\right) e_{u}(i) \\
& e_{u}(i+1)=\left(I_{m_{u}}-\Psi_{u}\left(i_{u}, e_{u}(i)\right)\right) e_{u}(i)
\end{aligned}
$$

when $q\left(i_{y}, i_{u}, e\right)=2$ and where $i+1=i_{y}+\left(i_{u}+1\right)$ (i.e., the counter $i_{u}$ is incremented by one and thus $i$ too). Based on (40)-(43), we thus have that the switching scheduling protocol is modeled by the protocol function $h_{s p}: \mathbb{N} \times \mathbb{N} \times \mathbb{R}^{m_{e}} \rightarrow \mathbb{R}^{m_{e}}$ that is given by

$$
\begin{aligned}
& h_{s p}\left(i_{y}, i_{u}, e\right)= \\
& \quad\left\{\begin{array}{l}
\left(h_{y}\left(i_{y}, e_{y}\right), e_{u}+D_{\mathbf{C}} w_{y}\right) \text { when } q\left(i_{y}, i_{u}, e\right)=1 \\
\left(e_{y}+D_{\mathbf{P}} w_{u}, h_{u}\left(i_{u}, e_{u}\right)\right) \text { when } q\left(i_{y}, i_{u}, e\right)=2,
\end{array}\right.
\end{aligned}
$$

for all $i_{y}, i_{u} \in \mathbb{N}$ and $e=\left(e_{y}, e_{u}\right) \in \mathbb{R}^{m_{e}}$ with $w_{y}:=$ $e_{y}-h_{y}\left(i_{y}, e_{y}\right)$ and $w_{u}:=e_{u}-h_{u}\left(i_{u}, e_{u}\right)$. Hence, such a switching protocol takes to some level the influence of the direct-feedthrough terms $D_{\mathbf{P}} / D_{\mathbf{C}}$ into account when deciding which node to update, leading to possible higher values of the MATI.

We will now analyze the stability properties of this novel protocol function for the system of (22) (which thus can be reformulated as (42)-(43)) for two specific switching laws $q$ that are based on the ideas of the RR and TOD protocols.

\subsubsection{Periodic switching scheduling protocol}

In this section, we introduce a switching law inspired by the idea of the RR protocol, i.e., we consider the case of periodically switching between updating the sensors and the actuators at each transmission instant. As such, we have that the switching law $q$ in this case is given by

$$
q\left(i_{y}, i_{u}, e\right)=q_{\mathrm{PS}}\left(i_{y}, i_{u}\right):=\left\{\begin{array}{l}
1, \text { if } i=1+2 j, j \in \mathbb{N} \\
2, \text { otherwise },
\end{array}\right.
$$

with $i=i_{y}+i_{u}$. For such a switching law, for which we coin the term periodic switching (PS) protocol, we formulate the following theorem.

Theorem 20 Let $D_{\mathbf{P}} \in \mathbb{R}^{m_{y} \times m_{u}}$ and $D_{\mathbf{C}} \in \mathbb{R}^{m_{u} \times m_{y}}$ be given. Assume there exist functions $W^{y}: \mathbb{N} \times \mathbb{R}^{m_{y}} \rightarrow \mathbb{R}_{\geq 0}$ and $W^{u}: \mathbb{N} \times \mathbb{R}^{m_{u}} \rightarrow \mathbb{R}_{\geq 0}$ that are globally Lipschitz in their second argument with Lipschitz constants $M^{y}$ and $M^{u}$, respectively, and suppose that the following holds:

(1) The scheduling protocol (function) $h_{y}: \mathbb{N} \times \mathbb{R}^{m_{y}} \rightarrow$ $\mathbb{R}^{m_{y}}$ is $(0,0)$-UGES with Lyapunov function $W^{y}$, i.e., the (nominal) discrete-time system

$$
e_{y}\left(i_{y}+1\right)=h_{y}\left(i_{y}, e_{y}\left(i_{y}\right)\right)
$$

is UGES with Lyapunov function $W^{y}$ in the sense that there exist positive lower and upper bounds $\underline{\alpha}_{W y}^{c}$ and $\bar{\alpha}_{W y}^{c}$, respectively, and a decreasing rate $\lambda^{y} \in[0,1)$ such that (18) holds for (46) with, hence, $p_{f}\left(i_{y}, e_{y}\right)=h_{y}\left(i_{y}, e_{y}\right)$. 
(2) The scheduling protocol (function) $h_{u}: \mathbb{N} \times \mathbb{R}^{m_{u}} \rightarrow$ $\mathbb{R}^{m_{u}}$ is $(0,0)$-UGES with Lyapunov function $W^{u}$, i.e., the (nominal) discrete-time system

$$
e_{u}\left(i_{u}+1\right)=h_{u}\left(i_{u}, e_{u}\left(i_{u}\right)\right)
$$

is UGES with Lyapunov function $W^{u}$ in the sense that there exist positive lower and upper bounds $\underline{\alpha}_{W u}^{c}$ and $\bar{\alpha}_{W^{u}}^{c}$, respectively, and a decreasing rate $\bar{\lambda}^{u} \in[0,1)$ such that (18) holds for (47) with, hence, $p_{f}\left(i_{u}, e_{u}\right)=h_{u}\left(i_{u}, e_{u}\right)$.

(3) It holds for some constants $\sigma>0$ and $\mu^{y}, \mu^{u}>1$ that

$\left|D_{\mathbf{C}}\right|<\frac{\underline{\alpha}_{W y}^{c}}{\sigma M^{u}}\left(1-\mu^{y} \lambda^{y}\right)$ and $\left|D_{\mathbf{P}}\right|<\frac{\sigma \underline{\alpha}_{W^{u}}^{c}}{M^{y}}\left(1-\mu^{u} \lambda^{u}\right)$.

Then, the PS protocol, modeled by $h_{s p}$ of (44) with the switching law $(45)$, is $\left(D_{\mathbf{P}}, D_{\mathbf{C}}\right)-U G E S$ with Lyapunov function $W: \mathbb{N} \times \mathbb{N} \times \mathbb{R}^{m_{e}} \rightarrow \mathbb{R}^{m_{e}}$, given by $W\left(i_{y}, i_{u}, e\right)=$

$$
\begin{cases}W^{y}\left(i_{y}, e_{y}\right)+\mu^{u} \sigma W^{u}\left(i_{u}, e_{u}\right), & q\left(i_{y}, i_{u}, e\right)=1 \\ \mu^{y} W^{y}\left(i_{y}, e_{y}\right)+\sigma W^{u}\left(i_{u}, e_{u}\right), & q\left(i_{y}, i_{u}, e\right)=2,\end{cases}
$$

where (18a) is satisfied for $\underline{\alpha}_{W}^{c} \leq \min \left\{\underline{\alpha}_{W y}^{c}, \sigma \underline{\alpha}_{W u}^{c}\right\}$ and $\bar{\alpha}_{W}^{c} \geq \max \left(\bar{\alpha}_{W^{y}}^{c}+\mu^{u} \sigma \bar{\alpha}_{W^{u}}^{c}, \mu^{y} \bar{\alpha}_{W^{y}}^{c}+\sigma \bar{\alpha}_{W^{u}}^{c}\right)$, (18b) for $\lambda=\max \left\{\frac{1}{\mu^{y}}, \frac{1}{\mu^{u}}, \mu^{y} \lambda^{y}+\alpha^{y}, \mu^{u} \lambda^{u}+\alpha^{u}\right\}$ where

$$
\alpha^{y}:=\frac{\sigma M^{u}}{\underline{\alpha}_{W^{y}}^{c}}\left|D_{\mathbf{C}}\right| \text { and } \alpha^{u}:=\frac{M^{y}}{\sigma \underline{\alpha}_{W^{u}}^{c}}\left|D_{\mathbf{P}}\right|,
$$

and (19) for $M=\max \left\{M^{y}+\mu^{u} \sigma M^{u}, \mu^{y} M^{y}+\sigma M^{u}\right\}$.

The proof is given in Appendix A. Observe now again, similar to Theorem 7, that the first two items in Theorem 20 merely require that the scheduling protocols for the sensors and actuators, respectively, are UGES in the classical sense of [23, Definition 7]. As such, we can thus implement different scheduling protocols for the sensors and actuators, respectively. Moreover, note that (48) is equivalent to the "small gain" type of condition

$$
\left|D_{\mathbf{C}}\right|\left|D_{\mathbf{P}}\right|<\frac{\underline{\alpha}_{W^{y}}^{c} \underline{\alpha}_{W^{u}}^{c}}{M^{u} M^{y}}\left(1-\lambda^{y}\right)\left(1-\lambda^{u}\right)
$$

since we can choose $\mu_{y}$ and $\mu_{u}$ arbitrarily close to one. Hence, for given matrices $D_{\mathbf{P}}$ and $D_{\mathbf{C}}$ satisfying (51) for chosen $(0,0)$-UGES protocol functions $h_{y}$ and $h_{u}$, there always exist $\sigma>0$ and $\mu_{y}, \mu_{u}>1$ such that the PS protocol is $\left(D_{\mathbf{P}}, D_{\mathbf{C}}\right)$-UGES. Moreover, when either one of the matrices $D_{\mathbf{P}}$ or $D_{\mathbf{C}}$ is absent (equal to the zero matrix), (51) is always satisfied and, hence, the other one's norm can grow arbitrarily large and still satisfy (48) (when the values of $\sigma$ and $\mu_{y}, \mu_{u}$ are chosen appropriately). As a consequence, when there is only one direct-feedthrough term present in the NCS, the PS protocol with (45) as the switching law always guarantees UGES of the error discrete-time system (and, hence, the NCS itself, under appropriate bounds on the MATI).

\subsubsection{Maximal error switching scheduling protocol}

Inspired by the idea behind the TOD protocol, we can also consider the scenario in which the switching law $q$ is determined by the values of the errors $e_{y}$ and $e_{u}$. In particular, we introduce a switching law, for which we coin the term maximal error switching (MES) protocol, where its value is determined by the Lyapunov functions $W^{y}$ and $W^{u}$ corresponding to the nominal systems (46) and (47), respectively, see items (1)-(2) of Theorem 20. That is, for some $\mu>0$, we introduce the switching law $q: \mathbb{N} \times \mathbb{N} \times \mathbb{R}^{m_{e}} \rightarrow\{1,2\}$ given by

$$
\begin{aligned}
& q\left(i_{y}, i_{u}, e\right)=q_{\operatorname{MES}}\left(i_{y}, i_{u}, e\right):= \\
& \left\{\begin{aligned}
\{1\}, \text { when } W^{y}\left(i_{y}, e_{y}\right)>\mu W^{u}\left(i_{u}, e_{u}\right) \\
\{2\}, \text { when } W^{y}\left(i_{y}, e_{y}\right)<\mu W^{u}\left(i_{u}, e_{u}\right) \\
\{1,2\}, \text { when } W^{y}\left(i_{y}, e_{y}\right)=\mu W^{u}\left(i_{u}, e_{u}\right) .
\end{aligned}\right.
\end{aligned}
$$

Now, similar to the PS protocol, we formulate the following theorem for the MES protocol.

Theorem 21 Let $D_{\mathbf{P}} \in \mathbb{R}^{m_{y} \times m_{u}}$ and $D_{\mathbf{C}} \in \mathbb{R}^{m_{u} \times m_{y}}$ be given. Assume there exist functions $W^{y}: \mathbb{N} \times \mathbb{R}^{m_{y}} \rightarrow \mathbb{R}_{\geq 0}$ and $W^{u}: \mathbb{N} \times \mathbb{R}^{m_{u}} \rightarrow \mathbb{R}_{\geq 0}$ that are globally Lipschitz in their second argument with Lipschitz constants $M^{y}$ and $M^{u}$, respectively, and suppose that the following hold:

(1) Items (1)-(2) of Theorem 20 are satisfied for constants $\underline{\alpha}_{W^{y}}^{c}, \bar{\alpha}_{W^{y}}^{c}, \underline{\alpha}_{W^{u}}^{c}, \bar{\alpha}_{W^{u}}^{c}>0$ and $\lambda^{y}, \lambda^{u} \in[0,1)$.

(2) It holds for some constant $\sigma>0$ that

$$
\left|D_{\mathbf{C}}\right|<\frac{\underline{\alpha}_{W^{y}}^{c}}{\sigma M^{u}}\left(1-\lambda^{y}\right) \text { and }\left|D_{\mathbf{P}}\right|<\frac{\sigma \underline{\alpha}_{W^{u}}^{c}}{M^{y}}\left(1-\lambda^{u}\right) .
$$

Then, the MES protocol, modeled by $h_{s p}$ of (44) with the switching law (52) for some $\mu>0$, is $\left(D_{\mathbf{P}}, D_{\mathbf{C}}\right)$-UGES with Lyapunov function $W: \mathbb{N} \times \mathbb{N} \times \mathbb{R}^{m_{e}} \rightarrow \mathbb{R}^{m_{e}}$, given by

$$
W\left(i_{y}, i_{u}, e\right)=W^{y}\left(i_{y}, e_{y}\right)+\sigma W^{u}\left(i_{u}, e_{u}\right),
$$

where (18a) is satisfied for $\underline{\alpha}_{W}^{c} \leq \min \left\{\underline{\alpha}_{W y}^{c}, \sigma \underline{\alpha}_{W}^{c}\right\}$ and $\bar{\alpha}_{W}^{c} \geq \bar{\alpha}_{W^{y}}^{c}+\sigma \bar{\alpha}_{W^{u}}^{c},(18 \mathrm{~b})$ for $\lambda=\max \left\{\lambda_{M E S}^{y}, \lambda_{M E S}^{u}\right\}$ with $\lambda_{M E S}^{y}:=\frac{\lambda^{y}+\alpha^{y}+\sigma \mu^{-1}}{1+\sigma \mu^{-1}}$ and $\lambda_{M E S}^{u}:=\frac{\lambda^{u}+\alpha^{u}+\sigma^{-1} \mu}{1+\sigma^{-1} \mu}$ and where $\alpha^{y}$ and $\alpha^{u}$ are given by (50), and (19) for $M=M^{y}+\sigma M^{u}$.

The proof is given in Appendix A. Observe that $\mu>0$ is a design parameter of the MES protocol, i.e., its value can be chosen in such a way to either give more priority to updating the sensors (small $\mu$ ) or the actuators (large $\mu$ ), which can be useful if the influence of either $D_{\mathbf{P}}$ or $D_{\mathbf{C}}$ on the error $e$ is much larger than that of the other one. Moreover, for a chosen value of $\mu>0$, one can maximize $\tau_{\text {mati }}$ over $\sigma>0$ subject to (53) and (20).

In addition, note that (53) is also equivalent to the small gain condition of (51), i.e., if (51) is satisfied, there always exists a $\sigma>0$ such that (53) is satisfied. This implies that the PS and MES protocols have the same condition under which they are guaranteed to be $\left(D_{\mathbf{P}}, D_{\mathbf{C}}\right)$ UGES. However, the computed values for $\underline{\alpha}_{W}^{c}, \bar{\alpha}_{W}^{c}$, and $\lambda$ (and, hence, the value for $\tau_{m a t i}$ ) differ. 


\section{Numerical example}

To illustrate the application of our results, we consider the benchmark example of the unstable batch reactor that can be captured by the model of (1)-(2) with

$$
\begin{aligned}
& A_{\mathbf{P}}=\left(\begin{array}{cccc}
1.38 & -0.208 & 6.715 & -5.676 \\
-0.5814 & -4.29 & 0 & 0.675 \\
1.067 & 4.273 & -6.654 & 5.893 \\
0.048 & 4.273 & 1.343 & -2.104
\end{array}\right) B_{\mathbf{P}}=\left(\begin{array}{cc}
0 & 0 \\
5.679 & 0 \\
1.136 & -3.146 \\
1.136 & 0
\end{array}\right)
\end{aligned}
$$

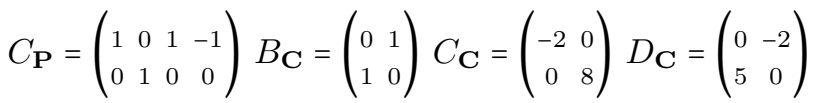

$A_{\mathbf{C}}=0$, and $D_{\mathbf{P}}=0$, see, e.g., $[5,14,16,23,30]$. Note that, since the matrix $D_{\mathbf{C}} \neq 0$, we indeed have directfeedthrough terms in the NCS. Here we assume, in contrast with $[5,14,23,30]$, that both the output $y$ to the controller as well as the controller output $u$ itself are transmitted over a communication network. As a result, the network-induced error is now updated according to (9), implying that the stability analysis of $[5,23]$ can no longer be applied. Fortunately, since $D_{\mathbf{P}}=0$, Assumption 1 is satisfied and, hence, we can apply Theorem 6 with any $\left(0, D_{\mathbf{C}}\right)$-UGES scheduling protocol to obtain a bound on the MATI and guarantee UGES for the NCS.

In [16], it has already been shown that exploiting the SD protocol guarantees (exponential) stability for the overall system, even with the error increasing at some transmission/inter-event times, see Fig. 4. To this end, Proposition 8 was used to compute the needed values for $\underline{\alpha}_{W}^{c}, \bar{\alpha}_{W}^{c}, M$, and $\lambda$, see Table 2 .

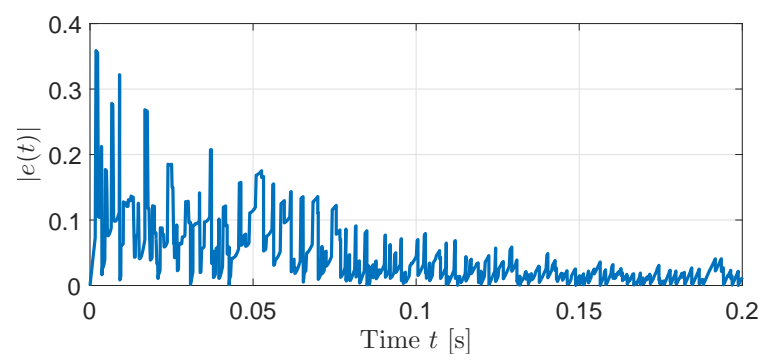

Fig. 4. Simulation results for the numerical example of the batch reactor with the SD protocol and $\tau_{\text {mati }}=1.840 \cdot 10^{-3}$.

In this paper, we compute also the bounds on the MATI using Theorem 6 for the RR protocol with $\ell=4$ (using Proposition 11), the SD+ protocol (using Proposition $17)$, and the MES protocol with $\ell_{y}=\ell_{u}=2, \mu=0.2$, and $\sigma=3.4315 \cdot 10^{-2}$ (using Theorem 21), where we implement the TOD protocol for the sensors $y$ and the RR protocol for the actuators $u$. Moreover, by simulating the NCS, we obtain numerical estimates of the actual bound $\tau_{\text {mati }}^{\text {sim }}$ on the MATI for which the NCS is still UGES. All the results are given in Table 2, from which we can draw various conclusions.

First, we can conclude that the SD+ protocol performance, as expected, significantly better than the standard SD protocol, proving that it indeed is useful to introduce this novel scheduling protocol function. Secondly however, we have that the computed $\tau_{\text {mati }}$ for the MES protocol is significantly lower than all the other computed bounds for the MATI, implying that its performance is worse than, for instance, that of the RR protocol, something that one would not expect. Indeed, when we simulate the NCS system for the various protocols, we obtain that the actual bound on the MATI $\tau_{\text {mati }}^{\text {sim }}$ for the MES protocol is larger than for the RR protocol, see Table 2. This difference in results can be explained by the fact that the conditions presented in Theorem 21 are only sufficient, whereas we have that Proposition 11 provides sufficient and necessary conditions. As such, similar to Theorem 7 , we can conclude that, while the conditions presented in Theorem 21 hold in general, they are also conservative. Improving upon these conditions is therefore a topic of future research. In particular, based on the observation that the error can increase when updating the networked values, exploiting, for instance, finite-step Lyapunov functions, see, e.g., [10], might result in less conservative results for all of the protocols.

Table 2

UGES scheduling protocols where $\tau_{m a t i}$ is computed using Theorem 6 and $\tau_{\text {mati }}^{\text {sim }}$ obtained through simulation.

\begin{tabular}{|c|c|c|c|c|c|}
\hline Prot. & $\underline{\alpha}_{W}^{c}$ & $\bar{\alpha}_{W}^{c} / M$ & $\lambda$ & $\tau_{\text {mati }}$ & $\tau_{\text {mati }}^{\text {sim }}$ \\
\hline $\mathrm{SD}$ & 0.120 & 1.409 & 0.424 & $1.840 \cdot 10^{-3}$ & 0.0626 \\
$\mathrm{RR}$ & 0.100 & 1.407 & 0.843 & $3.252 \cdot 10^{-4}$ & 0.0218 \\
$\mathrm{SD}+$ & 1 & 1 & 0 & $5.307 \cdot 10^{-2}$ & 0.1511 \\
$\mathrm{MES}$ & 0.034 & 1.049 & 0.957 & $3.856 \cdot 10^{-5}$ & 0.0455 \\
\hline
\end{tabular}

\section{Concluding remarks}

In this paper, we considered NCSs composed of a linear plant and linear controller with direct-feedthrough terms. Such terms are essential in the modeling of certain important classes of controllers commonly used in industry such as $\mathrm{PI}(\mathrm{D})$ regulators. To analyze stability of these "linear" NCSs, the concept of UGES scheduling protocols as introduced in [23] was extended to take into account the influence of the direct-feedthrough terms on the networked-induced error, resulting in the notion of $\left(D_{\mathbf{P}}, D_{\mathbf{C}}\right)$-UGES scheduling protocols with $D_{\mathbf{P}}$ and $D_{\mathbf{C}}$ being the direct-feedthrough terms in the plant and controller, respectively. We have shown that, under a well-posedness assumption, the stability analysis of [23] and [5], which resulted in conditions on the MATI for the NCS, can still be used as long as the protocols satisfy the $\left(D_{\mathbf{P}}, D_{\mathbf{C}}\right)$-UGES property. Therefore, we provided generic conditions on the direct-feedthrough terms $D_{\mathbf{P}}$ and $D_{\mathbf{C}}$ under which any UGES scheduling protocol in the classical sense is also $\left(D_{\mathbf{P}}, D_{\mathbf{C}}\right)$-UGES. Moreover, it has been shown that, for the well-known SD, RR, and TOD protocols, these conditions can be relaxed by exploiting the knowledge we have about the structure of the protocol. This analysis also revealed that using the "smarter" TOD protocol is not always better than the 
RR protocol and that updating all the nodes simultaneously (exploiting the SD protocol) can be worse than updating the nodes one by one. Furthermore, we also introduced new $\left(D_{\mathbf{P}}, D_{\mathbf{C}}\right)$-UGES scheduling protocols (i.e., the $\mathrm{SD}+, \mathrm{PS}$, and MES protocols) to handle the direct-feedthrough terms in a more effective way. Finally, we have illustrated the application of our new results by means of the benchmark example of the batch reactor and we compared various scheduling protocols.

The results presented in this paper for "linear" NCSs gave insights in the (counterintuitive) difficulties one encounters by including direct-feedthrough terms in the NCS setup. As such, the obtained results also have many natural extensions. Particularly, they are insightful for the nonlinear case, as, for instance, it should be possible to extend the general conditions as obtained in Theorem 7 to the nonlinear case by using Lipschitz properties. Moreover, the results in this paper could incite one to think of other new scheduling protocols, which exploit the available information about the direct-feedthrough terms better. In addition, the increase of the error norm after some time steps could encourage to investigate the use of finite-step Lyapunov functions in the context of NCSs with direct-feedthrough terms to obtain less conservative results.

\section{Appendices}

\section{A Proofs}

Proof of Theorem 7. Let $i \in \mathbb{N}, e \in \mathbb{R}^{m_{e}}$, and $w=$ $\Psi(i, e) e$. Addition to and subtraction from (18b) with $p_{f}(i, e)=h(i, e)$ of the term $W(i+1, h(i, e)+\mathbf{D} w)$, we obtain

$$
\begin{aligned}
& W(i+1, h(i, e)+\mathbf{D} w) \\
& \leq \lambda W(i, e)+W(i+1, h(i, e)+\mathbf{D} w)-W(i+1, h(i, e)) \\
& \leq \lambda W(i, e)+M|\mathbf{D} w| \leq \lambda W(i, e)+M|\mathbf{D}||w|
\end{aligned}
$$

where we used the Lipschitz property of $W$ in the second inequality. Since for the considered protocols it holds that $|w|=|\Psi(i, e) e| \leq|e|$ for all $e \in \mathbb{R}^{m_{e}}$ and by using (18a), we obtain that

$$
\begin{aligned}
W(i+1, h(i, e)+\mathbf{D} w) & \leq \lambda W(i, e)+M|\mathbf{D} \| e| \\
& \leq\left(\lambda+\left(\underline{\alpha}_{W}^{c}\right)^{-1} M|\mathbf{D}|\right) W(i, e),
\end{aligned}
$$$$
\text { which is equivalent to }
$$

$$
W(i+1, h(i, e)+\mathbf{D} w) \leq \rho W(i, e)
$$

for $\rho:=\lambda+\underline{\alpha}_{W}^{c}{ }^{-1} M|\mathbf{D}|$. Since (24) holds, we obtain that $\rho \in[0,1)$. This completes the proof.

Proof of Proposition 11. As a result of (29), it follows that the discrete-time system of (16) becomes a periodic linear discrete-time system of the form $e(i+1)=A_{k} e(i)$ with the matrix $A_{k}$ of $(32)$ and where $k=i \bmod \ell$. Hence, stability of the RR protocol can be analyzed by using the periodic Lyapunov Lemma, see, e.g., [2,3], which directly results in the LMI-based necessary and sufficient conditions of (31). This completes the proof.
Proof of Proposition 14. As mentioned above Proposition 14, since the resulting discrete-time system of (37) is a PWL system, $\left(D_{\mathbf{P}}, D_{\mathbf{C}}\right)$-UGES of the TOD protocol can be determined by solving a set of LMI's. In particular, to this end, we propose to use the S-procedure $[4,8,13]$. Consider hereto again the Lyapunov function $W(i, e)=\sqrt{e^{\top} P e}$ with $P$ being a symmetric positive definite matrix, and define for each $k \in \bar{\ell}$ the function

$$
S_{k}(e):=\sum_{j=1, j \neq k}^{\ell} \beta_{j}^{k} e^{\top} Q_{k j} e
$$

for the constants $\beta_{j}^{k} \geq 0, j \neq k$, such that $S_{k}(e) \geq 0$ when $e \in \mathcal{C}_{k}$. It follows that, in order for the discretetime system of (37) to be UGES, the following condition should be satisfied for all $i \in \mathbb{N}, e \in \mathbb{R}^{m_{e}}$, and $k \in \bar{\ell}$

$$
\begin{array}{r}
W^{2}\left(i+1, A_{k} e\right)-\rho W^{2}(i, e)+S_{k}(e) \leq 0 \\
\Rightarrow \quad e^{\top} A_{k}^{\top} P A_{k} e-\rho\left(e^{\top} P e\right)+\sum_{j \neq k} \beta_{j}^{k} e^{\top} Q_{k j} e \leq 0
\end{array}
$$

when $e \in \mathcal{C}_{k}$ with $0<\rho<1$. This leads directly to (38) and therefore completes the proof.

Proof of Theorem 20. Let $i_{y}, i_{u} \in \mathbb{N}, e=\left(e_{y}, e_{u}\right) \in$ $\mathbb{R}^{m_{e}}, i=i_{u}+i_{y}, w_{y}=e_{y}-h_{y}\left(i_{y}, e_{y}\right)$, and $w_{y}=$ $e_{u}-h_{u}\left(i_{u}, e_{u}\right)$. Consider the Lyapunov function for the overall system (42)-(43) with (45) given by (49) that clearly satisfies (18a) and (19) for any $\sigma>0$ and $\mu^{y}, \mu^{u}>1$, where $\underline{\alpha}_{W}^{c}, \bar{\alpha}_{W}^{c}$, and $M$ are given in the formulation of Theorem 20. Let now $q\left(i_{y}, i_{u}, e\right)=1$. In this case we have that the error dynamics are given by (42) and that $q\left(i_{y}+1, i_{u}, h_{s p}\left(i_{y}, i_{u}, e\right)\right)=2$. As such, it follows that

$$
\begin{aligned}
W & \left(i_{y}+1, i_{u},\left(h_{y}\left(i_{y}, e_{y}\right), e_{u}+D_{\mathbf{C}} w_{y}\right)\right) \\
= & \mu^{y} W^{y}\left(i_{y}+1, h_{y}\left(i_{y}, e_{y}\right)\right)+\sigma W^{u}\left(i_{u}, e_{u}+D_{\mathbf{C}} w_{y}\right) \\
= & \mu^{y} W^{y}\left(i_{y}+1, h_{y}\left(i_{y}, e_{y}\right)\right)+\sigma W^{u}\left(i_{u}, e_{u}+D_{\mathbf{C}} w_{y}\right) \\
& +\sigma\left(W^{u}\left(i_{u}, e_{u}\right)-W^{u}\left(i_{u}, e_{u}\right)\right) \\
\leq & \mu^{y} \lambda^{y} W^{y}\left(i_{y}, e_{y}\right)+\sigma W^{u}\left(i_{u}, e_{u}\right)+\sigma M^{u}\left|D_{\mathbf{C}} w_{y}\right| \\
\leq & \underbrace{\left(\mu^{y} \lambda^{y}+\frac{\sigma M^{u}}{\underline{\alpha}_{W y}^{c}}\left|D_{\mathbf{C}}\right|\right)}_{=: \tilde{\lambda}_{P S}^{y}} W^{y}\left(i_{y}, e_{y}\right)+\sigma W^{u}\left(i_{u}, e_{u}\right),
\end{aligned}
$$

where we used Lebourg's Lipschitz mean-value theorem [6, Theorem 2.3.7] and the property

$$
\left|w_{y}\right| \leq\left|e_{y}\right| \leq\left(\underline{\alpha}_{W y}^{c}\right)^{-1} W^{y}\left(i_{y}, e_{y}\right) .
$$

Hence, when (48) holds, i.e., $\tilde{\lambda}_{P S}^{y}<1$, we have that

$W\left(i_{y}+1, i_{u},\left(h_{y}\left(i_{y}, e_{y}\right), e_{u}+D_{\mathbf{C}} w_{y}\right)\right) \leq \bar{\lambda}_{P S}^{y} W\left(i_{y}, i_{u}, e\right)$ for $\bar{\lambda}_{P S}^{y}=\max \left\{\frac{1}{\mu^{u}}, \tilde{\lambda}_{P S}^{y}\right\} \in(0,1)$. Similarly, for the case that $q\left(i_{y}, i_{u}, e\right)=2$ (and, hence, $q\left(i_{y}, i_{u}+1, h_{s p}\left(i_{y}, i_{u}\right.\right.$, e) $)=1$, we obtain that 


$$
\begin{aligned}
& W\left(i_{y}, i_{u}+1,\left(e_{y}+D_{\mathbf{P}} w_{u}, h_{u}\left(i_{u}, e_{u}\right)\right)\right) \\
& \left.=W^{y}\left(i_{y}, e_{y}+D_{\mathbf{P}} w_{u}\right)\right)+\mu^{u} \sigma W^{u}\left(i_{u}+1, h_{u}\left(i_{u}, e_{u}\right)\right) \\
& \leq W^{y}\left(i_{y}, e_{y}\right)+\underbrace{\left(\mu^{u} \lambda^{u}+\frac{M^{y}}{\sigma \underline{\alpha}_{W}^{c}}\left|D_{\mathbf{P}}\right|\right)}_{=: \tilde{\lambda}_{P S}^{u}} \sigma W^{u}\left(i_{u}, e_{u}\right),
\end{aligned}
$$

implying that, when (48) holds, i.e., $\tilde{\lambda}_{P S}^{u}<1$, also

$W\left(i_{y}, i_{u}+1,\left(e_{y}+D_{\mathbf{P}} w_{u}, h_{u}\left(i_{u}, e_{u}\right)\right)\right) \leq \bar{\lambda}_{P S}^{u} W\left(i_{y}, i_{u}, e\right)$ for $\bar{\lambda}_{P S}^{u}=\max \left\{\frac{1}{\mu^{y}}, \tilde{\lambda}_{P S}^{u}\right\} \in(0,1)$. Based on the above results, it can thus be concluded that (with some abuse of notation) also (18b) is satisfied for the Lyapunov function (49) with $\lambda=\max \left\{\frac{1}{\mu^{y}}, \frac{1}{\mu^{u}}, \tilde{\lambda}_{P S}^{y}, \tilde{\lambda}_{P S}^{u}\right\}$. This completes the proof.

Proof of Theorem 21. Similarly to the proof of Theorem 20, let $i_{y}, i_{u} \in \mathbb{N}, e=\left(e_{y}, e_{u}\right) \in \mathbb{R}^{m_{e}}, i=i_{u}+i_{y}$, $w_{y}=e_{y}-h_{y}\left(i_{y}, e_{y}\right)$, and $w_{u}=e_{u}-h_{u}\left(i_{u}, e_{u}\right)$ and consider the Lyapunov function for the overall system (42)-(43) with (52) given by (54), which clearly satisfies (18a) and (19) for any constant $\sigma>0$, where $\underline{\alpha}_{W}^{c}, \bar{\alpha}_{W}^{c}$, and $M$ are given in the formulation of Theorem 21. Let now $q\left(i_{y}, i_{u}, e\right)=1$. In this case, the error dynamics are given by (42). As such, based on (A.1) it follows that

$$
\begin{aligned}
& W\left(i_{y}+1, i_{u},\left(h_{y}\left(i_{y}, e_{y}\right), e_{u}+D_{\mathbf{C}} w_{y}\right)\right) \\
& =W^{y}\left(i_{y}+1, h_{y}\left(i_{y}, e_{y}\right)\right)+\sigma W^{u}\left(i_{u}, e_{u}+D_{\mathbf{C}} w_{y}\right) \\
& \leq \underbrace{\left(\lambda^{y}+\frac{\sigma M^{u}}{\underline{\alpha}_{W^{y}}^{c}}\left|D_{\mathbf{C}}\right|\right)}_{:=\tilde{\lambda}_{M E S}^{y}} W^{y}\left(i_{y}, e_{y}\right)+\sigma W^{u}\left(i_{u}, e_{u}\right),
\end{aligned}
$$

Similarly, one obtains for the case $q\left(i_{y}, i_{u}, e\right)=2$ that

$$
\begin{aligned}
& W\left(i_{y}, i_{u}+1,\left(e_{y}+D_{\mathbf{P}} w_{u}, h_{u}\left(i_{u}, e_{u}\right)\right)\right) \\
& \leq W^{y}\left(i_{y}, e_{y}\right)+\underbrace{\left(\lambda^{u}+\frac{M^{y}}{\sigma \underline{\alpha}_{W u}^{c}}\left|D_{\mathbf{P}}\right|\right)}_{:=\tilde{\lambda}_{M E S}^{u}} \sigma W^{u}\left(i_{u}, e_{u}\right) .
\end{aligned}
$$

Note now that, when (53) is satisfied, we have

$$
\tilde{\lambda}_{M E S}^{y}<1 \text { and } \tilde{\lambda}_{M E S}^{u}<1 .
$$

To show now UGES of the system (42)-(43) for the MES protocol, we will make use of the properties induced by the switching law $q$ of (52). In particular, define the constants $0<\rho^{y}, \rho^{u}<1$ such that

$\tilde{\lambda}_{M E S}^{y}+\left(1-\rho^{y}\right) \sigma \mu^{-1}<1$ and $\tilde{\lambda}_{M E S}^{u}+\left(1-\rho^{u}\right) \sigma^{-1} \mu<1$,

which always exist as (A.4) holds (just take $\rho^{y}, \rho^{u}$ sufficiently close to one), and consider now the case of $q\left(i_{y}, i_{u}, e\right)=1$, i.e., $W^{y}\left(i_{y}, e_{y}\right) \geq \mu W^{u}\left(i_{u}, e_{u}\right)$. Based on (A.2), we have that

$$
\begin{aligned}
& W\left(i_{y}+1, i_{u}, h_{s p}\left(i_{y}, i_{u}, e\right)\right) \\
& \leq \tilde{\lambda}_{M E S}^{y} W^{y}\left(i_{y}, e_{y}\right)+\left(\rho^{y}+\left(1-\rho^{y}\right)\right) \sigma W^{u}\left(i_{u}, e_{u}\right) \\
& \stackrel{(52)}{\leq}\left(\tilde{\lambda}_{M E S}^{y}+\left(1-\rho^{y}\right) \sigma \mu^{-1}\right) W^{y}\left(i_{y}, e_{y}\right)+\rho^{y} \sigma W^{u}\left(i_{u}, e_{u}\right) \\
& \leq \underbrace{\max \left\{\rho^{y}, \tilde{\lambda}_{M E S}^{y}+\left(1-\rho^{y}\right) \sigma \mu^{-1}\right\}}_{=: \bar{\lambda}_{M E S}^{y} \in(0,1)} W\left(i_{y}, i_{u}, e\right) .
\end{aligned}
$$

Similarly, one can obtain that in the case of $q\left(i_{y}, i_{u}, e\right)=$ 2, i.e., $W^{y}\left(i_{y}, e_{y}\right) \leq \mu W^{u}\left(i_{u}, e_{u}\right)$, that (cf. (A.3))

$$
\begin{aligned}
& W\left(i_{y}, i_{u}+1, h_{s p}\left(i_{y}, i_{u}, e\right)\right) \\
& \leq\left(\rho^{u}+\left(1-\rho^{u}\right)\right) W^{y}\left(i_{y}, e_{y}\right)+\tilde{\lambda}_{M E S}^{u} \sigma W^{u}\left(i_{u}, e_{u}\right) \\
& \stackrel{(52))\left(\tilde{\lambda}_{M E S}^{u}+\left(1-\rho^{u}\right) \sigma^{-1} \mu\right) \sigma W^{u}\left(i_{u}, e_{u}\right)+\rho^{u} W^{y}\left(i_{y}, e_{y}\right)}{\leq} \underbrace{\max \left\{\rho^{u}, \tilde{\lambda}_{M E S}^{u}+\left(1-\rho^{u}\right) \sigma^{-1} \mu\right\}}_{=: \bar{\lambda}_{M E S}^{u} \in(0,1)} W\left(i_{y}, i_{u}, e\right) .
\end{aligned}
$$

This analysis shows that UGES of the system (42)-(43) is indeed guaranteed for the switching law of (52). In particular, we have that (with some abuse of notation) also (18b) is satisfied for the switching law of (52) with $\lambda=\max \left\{\bar{\lambda}_{M E S}^{y}, \bar{\lambda}_{M E S}^{u}\right\} \in(0,1)$. Moreover, observe that $\bar{\lambda}_{M E S}^{y}$ reaches its minimal value when $\rho^{y}=\left(\tilde{\lambda}_{M E S}^{y}+\right.$ $\left.\sigma \mu^{-1}\right)\left(1+\sigma \mu^{-1}\right)^{-1}$, and, similarly, $\bar{\lambda}_{M E S}^{u}$ reaches its minimal value for $\rho^{u}=\left(\tilde{\lambda}_{M E S}^{u}+\sigma^{-1} \mu\right)\left(1+\sigma^{-1} \mu\right)^{-1}$. As such, we have that $(18 \mathrm{~b})$ also holds with

$$
\lambda=\max \left\{\frac{\tilde{\lambda}_{M E S}^{y}+\sigma \mu^{-1}}{1+\sigma \mu^{-1}}, \frac{\tilde{\lambda}_{M E S}^{u}+\sigma^{-1} \mu}{1+\sigma^{-1} \mu}\right\} .
$$

This completes the proof.

\section{B Trajectory evolution for the RR protocol}

Consider the discrete-time system of (22) with (28) without any mixed nodes and the partitioned error vector

$$
\begin{aligned}
e(i) & =\left(e_{1}(i), \ldots, e_{\ell_{y}}(i), e_{\ell_{y}+1}(i), \ldots, e_{\ell_{y}+\ell_{u}}(i)\right) \\
& =\left(e_{y}(i), e_{u}(i)\right) .
\end{aligned}
$$

For the considered RR protocol, we can now compute the trajectory from any initial condition $e(0) \in \mathbb{R}^{m_{e}}$. To this end, we also partition the matrices $D_{\mathbf{P}}$ and $D_{\mathbf{C}}$ to reflect the structure of (B.1), i.e., we have that

$D_{\mathbf{P}}=\left[\begin{array}{ccc}D_{\mathbf{P}}^{11} & \cdots & D_{\mathbf{P}}^{1 \ell_{u}} \\ \vdots & \ddots & \vdots \\ D_{\mathbf{P}}^{\ell_{y} 1} & \cdots & D_{\mathbf{P}}^{\ell_{y} \ell_{u}}\end{array}\right]$ and $D_{\mathbf{C}}=\left[\begin{array}{ccc}D_{\mathbf{C}}^{11} & \cdots & D_{\mathbf{C}}^{1 \ell_{y}} \\ \vdots & \ddots & \vdots \\ D_{\mathbf{C}}^{\ell_{u} 1} & \cdots & D_{\mathbf{C}}^{\ell_{u} \ell_{y}}\end{array}\right]$.

Since the RR protocol is a $\ell$-periodic protocol, it suffices to compute the first $\ell$ steps of the trajectory. According to (29), we first have an update of the first node, i.e., in (28) we have that $\Delta(i)=\operatorname{diag}\left\{I_{m_{1}}, 0_{m_{2}}, \ldots, 0_{m_{\ell}}\right\}$, which implies that (22) for the first step results in 


$$
e(1)=\left(e_{y}(1), e_{u}(1)\right)
$$

$$
\begin{aligned}
& =\left[\begin{array}{c}
0 \\
e_{2}(0) \\
\vdots \\
e_{\ell_{y}}(0) \\
e_{\ell_{y}+1}(0) \\
\vdots \\
e_{\ell}(0)
\end{array}\right]+\left[\begin{array}{cccccc} 
& & & D_{\mathbf{P}}^{11} & \cdots & D_{\mathbf{P}}^{1 \ell_{u}} \\
& 0_{m_{y}} & & \vdots & \ddots & \vdots \\
& & & D_{\mathbf{P}}^{\ell_{y} 1} & \cdots & D_{\mathbf{P}}^{\ell_{y} \ell_{u}} \\
D_{\mathbf{C}}^{11} & \cdots & D_{\mathbf{C}}^{1 \ell_{y}} & & & \\
\vdots & \ddots & \vdots & & 0_{m_{u}} \\
D_{\mathbf{C}}^{\ell_{u}} 1 & \cdots & D_{\mathbf{C}}^{\ell_{u} \ell_{y}} & & &
\end{array}\right]\left[\begin{array}{c}
e_{1}(0) \\
0 \\
\vdots \\
0 \\
0 \\
\vdots \\
0
\end{array}\right] \\
& =\left(0, e_{2}(0), \cdots, e_{\ell_{y}}(0), e_{\ell_{y}+1}(0)+D_{\mathbf{C}}^{11} e_{1}(0)\right. \text {, } \\
& \left.\cdots, e_{\ell}(0)+D_{\mathbf{C}}^{\ell_{u} 1} e_{1}(0)\right)
\end{aligned}
$$

for any initial condition $e(0) \in \mathbb{R}^{m_{e}}$. Hence, one can see that an update of a sensor node does not influence the error vector $e_{y}$ (except for the node that is updated). As a result, repeating the update for all sensor nodes results in

$$
\begin{aligned}
e\left(\ell_{y}\right) & =\left[\begin{array}{c}
0 \\
\vdots \\
0 \\
e_{\ell_{y}+1}(0)+D_{\mathbf{C}}^{11} e_{1}(0)+\cdots+D_{\mathbf{C}}^{1 \ell_{y}} e_{\ell_{y}}(0) \\
\vdots \\
e_{\ell}(0)+D_{\mathbf{C}}^{\ell_{u} 1} e_{1}(0)+\cdots+D_{\mathbf{C}}^{\ell_{u} \ell_{y}} e_{\ell_{y}}(0)
\end{array}\right] \\
& =\left(0, e_{u}(0)+D_{\mathbf{C}} e_{y}(0)\right)=\left(e_{y}\left(l_{y}\right), e_{u}\left(l_{y}\right)\right) .
\end{aligned}
$$

Similarly, the next update corresponding to the first actuator node results in

$e\left(\ell_{y}+1\right)=$

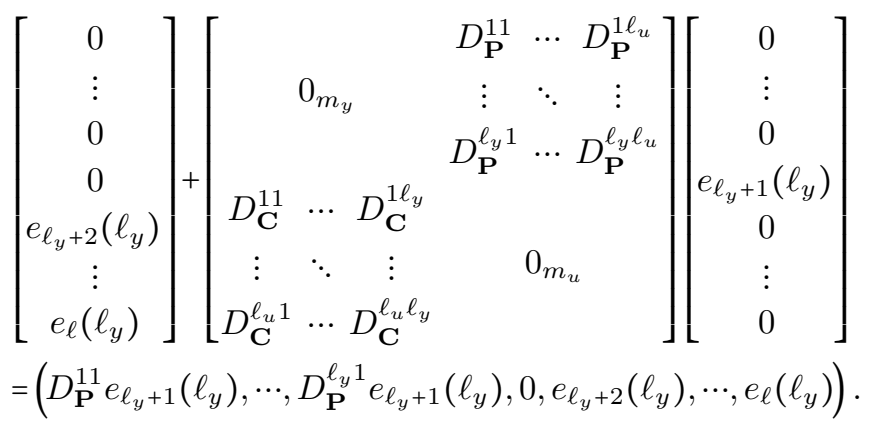

Hence, we can again observe that an update of an actuator node does not influence the error vector $e_{u}$ (except for the node that is updated). This implies that after all the actuator nodes have been updated, we have that

$$
\begin{aligned}
e(l) & =\left[\begin{array}{c}
D_{\mathbf{P}}^{11} e_{\ell_{y}+1}\left(\ell_{y}\right)+\cdots+D_{\mathbf{P}}^{1 \ell_{u}} e_{\ell}\left(\ell_{y}\right) \\
\vdots \\
D_{\mathbf{P}}^{\ell_{y} 1} e_{\ell_{y}+1}\left(\ell_{y}\right)+\cdots+D_{\mathbf{P}}^{\ell_{y} \ell_{u}} e_{\ell}\left(\ell_{y}\right) \\
0 \\
\vdots \\
0
\end{array}\right] . \\
= & {\left[\begin{array}{c}
D_{\mathbf{P}} e_{u}\left(\ell_{y}\right) \\
0
\end{array}\right]=\left[\begin{array}{c}
D_{\mathbf{P}} e_{u}(0)+D_{\mathbf{P}} D_{\mathbf{C}} e_{y}(0) \\
0
\end{array}\right] . }
\end{aligned}
$$

As such, it now directly follows that the evolution of the discrete-time system given by (22) with the RR protocol of (28) after $j \ell$ steps, $j \in \mathbb{N}$, is given by

$$
e(j \ell)=\left[\begin{array}{c}
\left(D_{\mathbf{P}} D_{\mathbf{C}}\right)^{j-1} D_{\mathbf{P}} e_{u}(0)+\left(D_{\mathbf{P}} D_{\mathbf{C}}\right)^{j} e_{y}(0) \\
\mathbf{0}_{m_{u}}
\end{array}\right],
$$

where $\mathbf{0}_{m_{u}}$ denotes the vector of all zeros of size $m_{u}$.

\section{References}

[1] D.S. Bernstein. Matrix Mathematics: Theory, Facts, and Formulas (Second Edition). Princeton reference. Princeton University Press, 2009.

[2] S. Bittanti, P. Bolzern, and P. Colaneri. Stability analysis of linear periodic systems via the Lyapunov equation. In Proceedings of the 10th IFAC World Congress, pages 169$172,1984$.

[3] P. Bolzern and P. Colaneri. The periodic Lyapunov equation. SIAM Journal of Matrix Analysis Application, (9):499-512, 1988.

[4] S. Boyd, L.E. Ghaoui, E. Feron, and V. Balakrishnan. Linear Matrix Inequalities in System and Control Theory. SIAM, 1993.

[5] D. Carnevale, A.R. Teel, and D. Nešić. A Lyapunov proof of an improved maximum allowable transfer interval for networked control systems. IEEE Transactions on Automatic Control, 52(5):892-897, 2007.

[6] F.H. Clarke. Optimization and nonsmooth analysis. Society for Industrial and Applied Mathematics, Philadelphia, Pa, 1990.

[7] V.S. Dolk, D.P. Borgers, and W.P.M.H. Heemels. Outputbased and decentralized dynamic event-triggered control with guaranteed $\mathcal{L}_{p}$-gain performance and Zeno-freeness. IEEE Transactions on Automatic Control, 62(1):34-49, 2017.

[8] G. Ferrari-Trecate, F.A. Cuzzola, D. Mignone, and M. Morari. Analysis of discrete-time piecewise affine and hybrid systems. Automatica, 38(12):2139-2146, 2002.

[9] H. Gao, T. Chen, and J. Lam. A new delay system approach to network-based control. Automatica, 44(1):39-52, 2008.

[10] R. Geiselhart, R.H. Gielen, M. Lazar, and F.R. Wirth. An alternative converse Lyapunov theorem for discrete-time systems. Systems \& Control Letters, 70:49-59, 2014.

[11] R. Goebel, R.G. Sanfelice, and A.R. Teel. Hybrid dynamical systems. Princeton University Press, United Kingdom, 2012.

[12] W.P.M.H. Heemels, D.P. Borgers, N. van de Wouw, D. Nešić, and A.R. Teel. Stability analysis of nonlinear networked control systems with asynchronous communication: A smallgain approach. In Proceedings of the IEEE 52 $2^{\text {nd }}$ Conference on Decision and Control, pages 4631-4637, 2013.

[13] W.P.M.H. Heemels, G.E. Dullerud, and A.R. Teel. $\mathcal{L}_{2}$-Gain analysis for a class of hybrid systems with applications to reset and event-triggered control: A lifting approach. IEEE Transactions on Automatic Control, 61(10):2766-2781, 2016.

[14] W.P.M.H. Heemels, A.R. Teel, N. van de Wouw, and D. Nešić. Networked control systems with communication constraints: Tradeoffs between transmission intervals, delays, and performance. IEEE Transactions on Automatic Control, 55(8):1781-1796, 2010. 
[15] S.H.J. Heijmans, D.P. Borgers, and W.P.M.H. Heemels. Stability and performance analysis of spatially invariant systems with networked communication. IEEE Transactions on Automatic Control, 62(10):4994-5009, 2017.

[16] S.H.J. Heijmans, R. Postoyan, D. Nešić, N. Noroozi, and W.P.M.H. Heemels. Stability analysis of networked control systems with direct-feedthrough terms: Part II - The linear case. In Proceedings of the IEEE $55^{\text {th }}$ Conference on Decision and Control, pages 5974-5979, 2016.

[17] S.H.J. Heijmans, R. Postoyan, D. Nešić, and W.P.M.H. Heemels. Computing minimal and maximal allowable transmission intervals for networked control systems using the hybrid systems approach. IEEE Control Systems Letters, 1(1):56-61, 2017.

[18] J.P. Hespanha, P. Naghshtabrizi, and Y. Xu. A survey of recent results in networked control systems. Proceedings of the IEEE, 95(1):138-162, 2007.

[19] H.K. Khalil. Nonlinear systems. Prentice-Hall, Inc., Upper Saddle River, New Jersey, third edition, 2002.

[20] A. Lamperski and A.D. Ames. Lyapunov theory for Zeno stability. IEEE Transactions on Automatic Control, 58(1):100-112, 2013.

[21] H. Lin and P.J. Antsaklis. Stability and stabilizability of switched linear systems: a survey of recent results. IEEE Transactions on Automatic control, 54(2):308-322, 2009.

[22] C. Meng, T. Wang, W. Chou, S. Luan, Y. Zhang, and Z. Tian. Remote surgery case: robot-assisted teleneurosurgery. In Proceedings of the IEEE International Conference on Robotics and Automation (ICRA'04), volume 1, pages 819823. IEEE, 2004.

[23] D. Nešić and A.R. Teel. Input-output stability properties of networked control systems. IEEE Transactions on Automatic Control, 49(10):1650-1667, 2004.

[24] N. Noroozi, R. Postoyan, D. Nešić, S.H.J. Heijmans, and W.P.M.H. Heemels. Stability analysis of networked control systems with direct-feedthrough terms: Part I - The nonlinear case. In Proceedings of the IEEE $55^{\text {th }}$ Conference on Decision and Control, pages 6820-6825, 2016.

[25] S. Öncü, N. van de Wouw, W.P.M.H. Heemels, and H. Nijmeijer. String stability of interconnected vehicles under communication constraints. In Proceedings of the IEEE $51^{\text {st }}$ Conference on Decision and Control, pages 2459-2464, 2012.

[26] R. Postoyan, N. van de Wouw, D. Nešić, and W.P.M.H. Heemels. Tracking control for nonlinear networked control systems. IEEE Transactions on Automatic Control, 59(6):1539-1554, 2014.

[27] R.S. Raji. Smart network for control. IEEE Spectrum, 31(6):49-55, 1994.

[28] R. Saeks. Resolution Space, Operators and Systems. Lecture Notes in Economics and Mathematical Systems, SpringerVerlag, Berlin, Germany, 1973.

[29] G.W. Stewart. Matrix Algorithms: Volume 1: Basic Decompositions. Society for Industrial and Applied Mathematics (SIAM), Philadelphia, United States of America, 1998.

[30] G.C. Walsh, H. Ye, and L.G. Bushnell. Stability analysis of networked control systems. IEEE Transactions on Control Systems Technology, 10(3):438-446, 2002.

[31] J.C. Willems. The analysis of feedback systems. The MIT Press, Cambridge, Massachusetts, 1971.

[32] T.C. Yang. Networked control system: A brief survey. IEEE Proceedings Control Theory and Applications, 153(4):403412, 2006.
[33] F. Zhang. The Schur Complement and Its Applications. Numerical Methods and Algorithms. Springer US, 2006.

[34] W. Zhang, M.S. Branicky, and S.M. Phillips. Stability of networked control systems. IEEE Control Systems, 21(1):8499, 2001.

[35] K. Zhou, J.C. Doyle, and K. Glover. Robust and optimal control, volume 40. Prentice hall New Jersey, 1996. 